\title{
Rectangular Layouts and Contact Graphs
}

\author{
Adam L. Buchsbaum* $\quad$ Emden R. Gansner* Cecilia M. Procopiuc* \\ Suresh Venkatasubramanian*
}

October 11, 2006

\begin{abstract}
Contact graphs of isothetic rectangles unify many concepts from applications including VLSI and architectural design, computational geometry, and GIS. Minimizing the area of their corresponding rectangular layouts is a key problem. We study the area-optimization problem and show that it is NP-hard to find a minimum-area rectangular layout of a given contact graph. We present $O(n)$-time algorithms that construct $O\left(n^{2}\right)$-area rectangular layouts for general contact graphs and $O(n \log n)$-area rectangular layouts for trees. (For trees, this is an $O(\log n)$-approximation algorithm.) We also present an infinite family of graphs (rsp., trees) that require $\Omega\left(n^{2}\right)$ (rsp., $\Omega(n \log n)$ ) area.

We derive these results by presenting a new characterization of graphs that admit rectangular layouts using the related concept of rectangular duals. A corollary to our results relates the class of graphs that admit rectangular layouts to rectangle of influence drawings.
\end{abstract}

\section{Introduction}

Given a set of objects in some space, the associated contact graph contains a vertex for each object and an edge implied by each pair of objects that touch in some prescribed fashion. While contact graphs have been extensively studied for objects such as curves, line segments, and even strings (surveyed by Hliněný [17] and Hliněný and Kratochvíl [18]), as has the more general class of intersection graphs (surveyed by Brandstädt, Le, and Spinrad [8] and McKee and McMorris [28]), the literature on closed shapes is relatively sparse. Koebe's Theorem [21] states that any planar graph (and only a planar graph) can be expressed as the contact graph of disks in the plane.1] More recently, de Fraysseix, Ossona de Mendez and Rosenstiehl [10] show that any planar graph can be represented as a triangle contact graph but not vice-versa. In this paper, we consider contact graphs of isothetic rectangles.

Contact graphs of rectangles find critical applications in areas including VLSI design [24, 44-46, 55], architectural design [42], and, in other formulations, computational geometry [9,31] and geographic information systems [12]. Previous works considered concepts similar to contact graphs but used a variety of notions like rectangular duality $[15,22,23,25]$ and concepts from graph drawing such as orthogonal, rectilinear, visibility, and proximity layouts $[3,4,38]$ as well as rectangular drawings [16,33-35] to achieve their results. Our work is the first to deal directly with contact graphs of rectangles, yielding a simpler foundation for study.

We call a collection of rectangles that realizes a given contact graph $G$ a rectangular layout: a set of disjoint, isothetic rectangles corresponding to the vertices of $G$, such that two rectangles are adjacent

\footnotetext{
*AT\&T Labs, Shannon Laboratory, 180 Park Ave., Florham Park, NJ 07932, \{alb,erg,magda,suresh\}@ research.att.com.

${ }^{1}$ This result was lost and recently rediscovered independently by Andreev and Thurston; Sachs [39] provides a history.
} 
if and only if the implied edge exists in $G$. The associated optimization problem is to find a rectangular layout minimizing some criterion such as area, width, or height. This problem is inherently intriguing, with many enticing subproblems and variations. It arises in practice in the design of an interface to a relational database system used in AT\&T to allow customers to model and administer the equipment and accounts in their telecommunications networks. This system allows users to specify their own schemas using entityrelationship models [36]. The system then presents the database entities as rectangular buttons. Clicking on a button provides information related to the corresponding entity type: e.g., detailed descriptions of the entity attributes or information about specific records. Experience indicates the benefit of juxtaposing buttons that correspond to related entities. Viewing the database schema as the obvious graph, with entities as vertices and relations as edges, leads to rectangular layouts. Solving the related optimization problems would automate this part of tailoring the interface to the schema, of significant benefit as otherwise each customer's interface must be built manually.

We solve several important problems concerning rectangular layouts. We give a new characterization of planar graphs that admit rectangular layouts in terms of those that can be embedded without filled triangles. En route, we unify a number of different lines of research in this field. We show a suite of results concerning the hardness of finding optimal layouts; design algorithms to construct layouts on graphs, and, with better area bounds, trees; and present some worst-case area lower bounds. We detail these results at the end of this section.

\subsection{Relationship to Prior Work}

Rectangular layouts are dual concepts to rectangle drawings of planar graphs: that is, straight-line, isothetic embeddings with only rectangular faces. Recent work on rectangular drawings [34,35] and the related boxrectangular drawings $[16,33]$ culminates with Rahman, Nishizeki, and Ghosh's linear-time algorithm for finding a rectangular drawing of any planar graph if one exists [35]. In a rectangular layout, the rectangles themselves correspond to vertices. While the two concepts are more or less dual to each other, moving between them can be highly technical. In particular, the machinery to find rectangular drawings of graphs that are not three-connected is complex. Our contribution gives a direct method for constructing rectangular layouts, and we handle cases of low connectivity easily.

Rectangular layouts are closely related to rectangular duals, which are like layouts except that a rectangular dual must form a dissection of its enclosing rectangle; i.e., it allows no gaps between rectangles. Rectangular duals have a rich history, including much work on characterizing graphs that admit rectangular duals $[15,22,23,25]$, transforming those that do not by adding new vertices [1,24], and constructing rectangular duals in linear time [5, 15,20]. The proscription of gaps, however, severely limits the class of graphs that admit rectangular duals; for example, paths are the only trees that have such duals. In general, any (necessarily planar) graph admitting a rectangular dual must be internally triangulated, but no such restriction applies to layouts. This simple distinction yields many advantages to layouts over duals. The cleaner, less specified definition of layouts characterizes a class of graphs that is both more general (including all trees, for example) and also much simpler to formalize. When we discuss area, we will also show that while different variations of rectangular layouts have different degrees of area monotonicity under graph augmentation, rectangular duals do not enjoy any such monotonicity: a small graph might require a significantly larger dual than a larger graph. Additionally, there are graphs that admit asymptotically smaller rectangular layouts than duals.

Another closely related area concerns VLSI floorplanning, in which an initial configuration of rectangles (usually a dissection) is given, the goal being to rearrange and resize the rectangles to minimize the area while preserving some properties of the original layout. While there is an extensive literature on floor- 
planning, there is much divergence within it as to what criteria must be preserved during minimization. These typically include minimum size constraints on the rectangles plus one of various notions of adjacency equivalence. Stockmeyer [43] presents one of the cleanest definitions of equivalence, preserving the notion of relative placement of rectangles (whether one appears left of, right of, above, or below another); other criteria include the preservation of relative area and aspect ratios [53] and that of relative lengths of abutment [46]. These works are further classified by sliceability: a floorplan is sliceable if it can be recursively deconstructed by vertical and horizontal lines extending fully across the bounding box. Minimizing the area of non-sliceable floorplans has been shown to be NP-hard under various constraints [26,32,43], whereas area minimization of sliceable floorplans is tractable $[41,43,54]$. Not all floorplans can be realized by sliceable equivalents [44,45], so work exists on isolating and generating sliceable floorplans where possible [55] as well as minimizing the area of non-sliceable floorplans by various heuristics $[32,46,53]$.

Two major facets distinguish floorplanning from area-minimization of rectangular layouts. First, floorplanning seeks to minimize the area of an arrangement of rectangles given a priori by some external process (possibly human design); rectangular layouts themselves are determined by corresponding contact graphs. Second, the notion of equivalence among floorplans differs from context to context, whereas contact graph adjacencies strictly identify the equivalence of rectangular layouts. Thus, there can exist different rectangular layouts of the same graph that do not represent equivalent floorplans, even by Stockmeyer's definition; and conversely there can exist equivalent floorplans that are layouts for non-isomorphic graphs. Still, much work in floorplanning uses concepts from rectangular duals, so work on rectangular layouts can also contribute to this area.

Finally, also related is the idea of proximity drawings $[4,19]$, in which a set of objects corresponds to the vertices of a graph, with edges connecting vertices of correspondingly close objects for some definition of proximity. A particularly relevant special case is that of rectangle of influence drawings [27], which are (not necessarily planar) straight-line embeddings of graphs such that the isothetic rectangles induced by pairs of vertices contain no other vertices if and only if the corresponding edges exist. Using results of Biedl, Bretscher, and Meijer [6], we show that graphs that admit rectangular layouts are precisely those that admit a weaker variation of planar rectangle of influence drawings, in which induced rectangles may be empty even if the corresponding edges are missing from the graph; i.e., that contact graphs of rectangles also express this variation of rectangle of influence drawings.

\subsection{Our Results}

The many parallel lines of research (in different communities) in the general area of contact graphs of rectangles have led to overlapping (and in some cases equivalent) definitions and results. Our contributions in this paper are two-fold: we present algorithms for optimizing rectangular layouts and prove various hardness results, and we also prove various structural results that tie existing work together in a coherent way that produces efficient algorithms.

- We provide a new characterization of the class of graphs that admit rectangular layouts. This characterization is equivalent to an earlier result by Thomassen [49] and has the added advantage of yielding an $O(n)$-time algorithm for checking if a given $n$-vertex planar graph admits such a layout. Moreover, we can construct a layout for the input graph in $O(n)$ time. We also prove an upper bound of $O\left(n^{2}\right)$ on the area of the layout, and we give a matching worst-case lower bound.

- We give an $O(n)$-time algorithm that constructs an $O(n \log n)$-area layout for any tree. We also demonstrate a general class of trees that are flexible: i.e., they can be laid out in linear area with any 
aspect ratio. Finally we show that in general trees cannot have arbitrarily thin layouts: there exists a class of trees such that the minimum dimension must have size $\Omega(\log n)$. This bound uses solely topological arguments and may be of independent interest. In particular, it leads to an $\Omega(n \log n)$ worst-case area lower bound, matching the upper bound of our algorithm.

- We prove that the problem of optimizing the area of a rectangular layout is NP-hard. The proof also shows that optimizing the width given a fixed height, or vice-versa, is NP-hard.

- We show that rectangular duals can be much larger than layouts; there exists a class of graphs having $O(n)$-area layouts but $\Omega\left(n^{2}\right)$-area duals.

- Our characterization of contact graphs of rectangles in terms of filled triangles establishes a connection between rectangular layouts and rectangle of influence drawings. Specifically, a corollary of our characterization is that the class of graphs having rectangular layouts is identical to the class of graphs having planar, weak, closed rectangle of influence drawings [6].

Paper Outline. We continue in Section 2 by introducing necessary definitions, including those for rectangular layouts themselves, and we also state some useful lemmas. In Section 3 we present our new characterization of graphs that admit rectangular layouts. We use this characterization in Section 4 to design our linear-time algorithm for constructing $O\left(n^{2}\right)$-area rectangular layouts, and we show matching worst-case lower bounds. In Section 5 we present improved results for trees. We present hardness results in Section 6 and we conclude in Section 7

\section{Definitions}

Throughout, we assume without loss of generality that all graphs are connected and have at least 4 vertices. For a given graph $G=(V, E)$, define $n=|V|$ and $m=|E|$. We say $G^{\prime}=\left(V^{\prime}, E^{\prime}\right)$ is a subgraph of $G$ if $V^{\prime} \subseteq V$ and $E^{\prime} \subseteq E$; $G^{\prime}$ is a proper subgraph of $G$ if $G^{\prime}$ is a subgraph of $G$ and $G^{\prime} \neq G$.

A graph $G$ is $k$-connected if the removal of any set of $k-1$ vertices leaves the remainder of $G$ connected. A separating triangle of $G$ is a 3-vertex cycle whose removal disconnects the remainder of $G$. For an arbitrary but fixed embedding of a planar graph, a filled triangle is defined to be a length-3 cycle with at least one vertex inside the induced region. Note that an embedding of a graph might have a filled triangle but no separating triangle (e.g., any embedding of $K_{4}$ ) and vice-versa. A cubic graph is a regular degree-3 graph. A planar triangulation is a planar graph in which all faces are bounded by 3-vertex cycles. Whitney's 2-Isomorphism Theorem [52] in fact implies that a planar triangulation has a unique embedding up to stereographic projection, which preserves the facial structure, so it is well-defined to talk about a planar graph $G$ itself being a triangulation (or triangulated) as opposed to some specific embedding of $G$ being triangulated. Note also that a planar triangulation $G$ has a planar dual, denoted $G^{*}$, which is unique up to isomorphism. Clearly, any 4-connected graph has no separating triangle; for planar triangulations, the converse holds as well.

Lemma 2.1 A planar triangulation $G$ is 4-connected if and only if $G$ has no separating triangles.

Koźmiński and Kinnen [22, Lem. 1] state Lemma 2.1 in terms of a fixed embedding, but the above remarks obviate the issue of fixed embeddings for planar triangulations.

A graph $G$ is cyclically $k$-edge connected if the removal of any $k-1$ edges either leaves $G$ connected or else produces at least one connected component that contains no cycle, i.e., does not break $G$ into multiple 

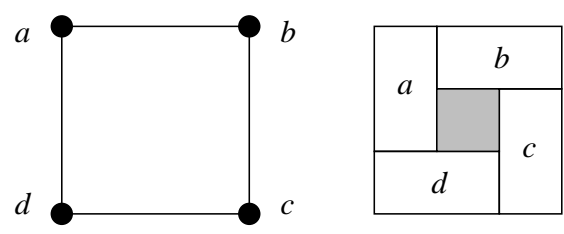

(a)

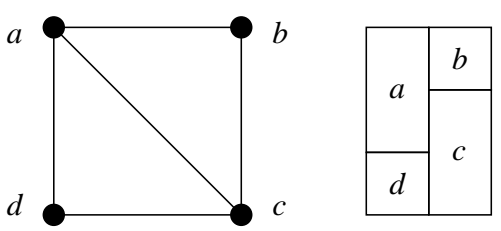

(b)

Figure 1: Two graphs and associated rectangular layouts. Shaded regions depict gaps.

connected components all of which contain cycles [51]. Cyclic 4-edge connectivity is a dual concept to 4-connectivity for planar triangulations. In particular, we will use the following result, which appears to be well known; we prove it in Appendix A for completeness.

Lemma 2.2 A graph $G$ is a 4-connected planar triangulation if and only if $G^{*}$ is planar, cubic, and cyclically 4-edge connected.

Define a rectangular dissection to be a partition of a rectangle into smaller rectangles, no four of which meet in any point. A rectangular representation of a graph $G$ is a rectangular dissection $R$ such that the vertices of $G$ map 1-to-1 to the intersections of line segments of $R$ minus the four external corners of $R$; i.e., $R$ is a straight-line, isothetic drawing of $G$ except for the four edges that form right angles at the external corners of $R$. A rectangular dual of a graph $G$ is a rectangular dissection, if one exists, whose geometric dual minus the exterior vertex and incident edges is $G$.

Rectangular duals allow no gaps; i.e., areas within the bounding box that do not correspond to any vertices. We introduce the concept of rectangular layout to allow gaps in the representation. This allows a larger class of graphs to be represented.

Definition 2.3 (Rectangular Layout) $A$ (strong) rectangular layout of a graph $G=(V, E)$ is a set $R$ of isothetic rectangles whose interiors are pairwise disjoint, with an isomorphism $\mathcal{R}: V \rightarrow R$ such that for any two vertices $u, v \in V$, the boundaries of $\mathcal{R}(u)$ and $\mathcal{R}(v)$ overlap non-trivially if and only if $\{u, v\} \in E$.

See Fig. 1. The requirement that non-trivial boundary overlaps define adjacencies (that rectangles meeting at corners are not considered adjacent) is significant: allowing corner-touching to imply adjacency changes the class of graphs expressed; cf., Section 7 This therefore obviates the specification that the rectangles be isothetic: any collection of rectangles inducing a connected contact graph must be isothetic. In contrast, there is no a priori proscription on trivial corner touching in layouts themselves. We shall show, however, that we can exclude such trivial corner touching without loss of generality.

We also define a relaxed variation: A weak rectangular layout of $G$ is a set $R$ of isothetic rectangles whose interiors are pairwise disjoint, with an isomorphism $\mathcal{R}: V \rightarrow R$ such that for any edge $\{u, v\} \in E$, the boundaries of $\mathcal{R}(u)$ and $\mathcal{R}(v)$ overlap non-trivially. In a weak layout, two rectangle boundaries may overlap even if the corresponding vertices are not adjacent. For example, in Fig. 1, layout (b) is a weak layout for both graphs (a) and (b) but a strong layout only for graph (b).

Consider a layout drawn on the integral grid. The area of the layout is that of the smallest enclosing isothetic bounding box. While corresponding weak and strong layouts may have different areas, from a feasibility standpoint the distinction does not matter, as shown by the following lemma. (Clearly any strong layout is also a weak layout.) 


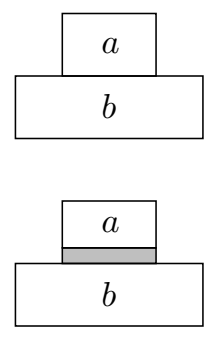

(a)

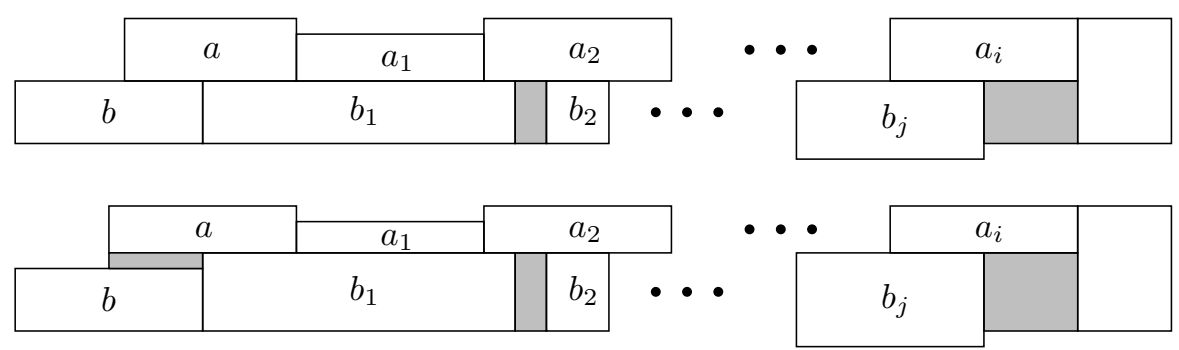

(b)

Figure 2: Weak layouts (top) and corresponding strong layouts (bottom) after fixing the $\{a, b\}$ violation. (a) The violating boundary of $a$ is nested in that of $b$. (b) No nesting occurs; shear the projection of the violating boundary until hitting a blocking rectangle. In both cases, there can be other rectangles not shown, but if the new separation $\epsilon$ is less than the smallest prior boundary overlap, no other adjacencies are broken.

Lemma 2.4 If $G$ has a weak rectangular layout $R$, then $G$ has some strong rectangular layout $L$.

Proof. Consider any two rectangles $a$ and $b$ in $R$ whose boundaries overlap but such that $\{a, b\} \notin E$. If the overlapping boundary of one (say $a$ ) is completely nested in that of the other $(b)$, we can separate the two rectangles by moving the touching boundary of $a$ away from $b$ by some small amount, $\epsilon$, that does not affect any other rectangle adjacency. Formally, let $\epsilon^{\prime}$ be the size of the smallest boundary overlap in $R$; then let $\epsilon=\epsilon^{\prime} / 2$. (On the integral grid, first scale all coordinates by a factor of 2, and then $\epsilon=1$.) Assume $a$ 's bottom boundary nests within $b$ 's top boundary; other cases are symmetric. Move $a$ 's bottom boundary up by $\epsilon$, leaving its other boundaries untouched; that is, $a$ is shrunk, not translated. See Figure 2 a). By construction of $\epsilon$, any rectangle touching $a$ on either its left or right side still touches $a$, and $a$ 's top boundary remains unchanged, so the only rectangle adjacency that is broken is that between $a$ and $b$.

If neither violating boundary is nested in the other, assume $a$ is to the right and top of $b$; other cases are symmetric. Consider the projection of the violating boundary rightwards until it hits some other rectangle or the right side of the bounding box. In left-to-right order, call the rectangles with bottom boundaries along this projection $a_{1}, a_{2}, \ldots, a_{i}$; and call the rectangles with top boundaries along this projection $b_{1}, b_{2}, \ldots, b_{j}$. See Figure 2(b). (Note there may be gaps along this projection.) Move the bottom boundaries of $a, a_{1}, a_{2}, \ldots, a_{i}$ and the top boundaries of $b_{1}, b_{2}, \ldots, b_{j}$ up by $\epsilon$ (as defined above). This fixes the $\{a, b\}$ violation. Furthermore, any rectangle adjacent to the left side of $a$ remains so by definition of $\epsilon$. Other boundary relationships among the adjusted rectangles are preserved, and no other boundaries are affected.

Repeating this process to fix each violation produces $L$.

Lemma 2.4 addresses feasibility of rectangle layouts only; the expansion in area from the transformation might be exponential. Later we give procedures to draw strong layouts directly with better areas.

In the sequel, we will use the term layout to refer to strong layouts. Furthermore, the assumption that no two rectangles meet trivially at a corner is without loss of generality. Say rectangles $a$ and $b$ so meet. We can perturb the boundaries by some small amount to make the boundary overlap non-trivial. The layout becomes weak if it was not already. Lemma 2.4 shows that it can be made strong with only non-trivial boundary overlaps. 


\section{Characterizing Rectangular Layouts}

\subsection{Background}

Thomassen characterizes graphs that admit rectangular layouts (which he calls strict rectangle graphs) as precisely the class of proper subgraphs of 4-connected planar triangulations [49, Thm. 2.1]. Together with earlier work [48] his work yields a polynomial-time algorithm for testing a graph $G$ to see if it admits a rectangular layout and, if so, constructing such a layout. He does not analyze the algorithm precisely for running time, however, nor does he bound the layout area at all, two criteria that concern us.

Thomassen's work rests critically on earlier work by Ungar [50]. Ungar defines a saturated plane map to be a finite set of non-overlapping regions that partitions the plane and satisfies the following conditions.

1. Precisely one region is infinite.

2. At most three regions meet in any point.

3. Every region is simply connected.

4. The union of any two adjacent regions is simply connected.

5. The intersection of any two regions is either a simple arc or is empty.

An $n$-ring is a set of $n$ regions such that their union is multiply connected 2 Ungar [50, Thm. A] shows that a saturated plane map that contains no 3-ring is isomorphic to a rectangular dissection. Ungar's Theorem B [50] further implies that for any rectangular dissection $R$ and any two adjacent rectangles $a$ and $b$ in $R$, there exists a rectangular dissection $R^{\prime}$ isomorphic to $R$ such that $a^{\prime}$ (the region in $R^{\prime}$ corresponding to $a$ in $R$ ) is the infinite region and $b^{\prime}$ (the rectangle in $R^{\prime}$ corresponding to $b$ in $R$ ) has three or four whole sides fully exposed: i.e., not overlapping any rectangle other than $a^{\prime}$.

We prove the following "folklore" lemma in Appendix A

Lemma 3.1 A graph $G$ is planar, cubic, and cyclically 4-edge connected if and only if $G$ is a saturated plane map with no 3-ring.

Using Lemma 3.1, Thomassen [49] rephrases Ungar's Theorem A as follows.

Lemma 3.2 Any cubic, cyclically 4-edge connected planar graph has a rectangular representation.

\subsection{New Characterization}

Koźmiński and Kinnen [22] define a 4-triangulation to be a 4-connected, planar triangulated graph with at least 6 vertices, at least one of which has degree 4. They prove [22, Thm. 1] that a cube with one face that is a rectangular dissection is dual to a planar graph $G$ if and only if $G$ is a 4-triangulation. They also prove [22, Thm. 2] that a planar graph $G$ with all faces triangular except the outside has a rectangular dual if and only if $G$ can be obtained from some 4-triangulation $H$ by the deletion of some degree-4 vertex and all its neighbors. We use the following somewhat weaker result to design an algorithm for constructing rectangular layouts.

Theorem 3.3 If a planar graph $G$ can be derived from some 4-connected planar triangulation $H$ by the removal of some vertex and its incident edges, then $G$ has a rectangular dual.

\footnotetext{
${ }^{2}$ Connectivity in this definition and conditions 35 above is in the topological sense.
} 
Proof. Let $H$ be any 4-connected planar triangulation and $v$ any vertex of $H$. By Lemma 2.2, $H^{*}$ is planar, cubic, and cyclically 4-edge connected, and so by Lemma $3.1 H^{*}$ yields a saturated plane map $M$ with no 3-ring. Ungar's Theorem B [50] implies that an isomorphic map $M^{\prime}$ exists with the external face corresponding to $v . M^{\prime}$ is thus a rectangular dual for the graph derived from $H$ by removing $v$ and its incident edges.

The benefit of Theorem 3.3 is that it gives a sufficient condition for rectangular duality in terms of 4connected triangulations rather than Koźmiński and Kinnen's [22] 4-triangulations. This yields (in Section 4) a simple augmentation procedure for constructing rectangular layouts based on the following alternative characterization of graphs admitting rectangular layouts.

Theorem 3.4 A planar graph $G$ is a proper subgraph of a 4-connected planar triangulation if and only if $G$ has an embedding with no filled triangles.

Proof. ( $\Longrightarrow$ ) Let $G^{\prime}$ be a 4-connected planar triangulation. Let $G$ be the result of removing any one edge or vertex (and its incident edges) from $G^{\prime}$. By Lemma 2.1, $G^{\prime}$, and therefore $G$, has no separating triangle. We claim that any embedding $\mathcal{E}$ of $G$ has a non-triangular face or that $G$ is itself just a 3-vertex cycle. To prove the claim, consider an arbitrary embedding $\mathcal{E}^{\prime}$ of $G^{\prime}$. Removing a single edge from $\mathcal{E}^{\prime}$ yields an embedding $\mathcal{E}$ (of $G$ ) with a non-triangular face. If removing a vertex $v$ from $\mathcal{E}^{\prime}$ yields an embedding $\mathcal{E}$ with no nontriangular face, then $\mathcal{E}$ itself must be a simple triangle; otherwise, the triangular face of $\mathcal{E}$ to which $v$ was adjacent is a separating triangle in $\mathcal{E}^{\prime}$, which by assumption cannot exist. This proves the claim.

If $G$ is a 3-vertex cycle, we are done; otherwise, by stereographic projection, we can assume that its external face is non-triangular. Because any filled triangle is either a separating triangle or the external face, it follows that $\mathcal{E}$ has no filled triangles, and therefore any proper subgraph of $G$ also has an embedding with no filled triangles.

$(\Longleftarrow$ ) Now let $G$ be a planar graph, and let $\mathcal{E}$ be some embedding of $G$ with no filled triangles. Assume without loss of generality that $G$ has at least one non-triangular face. Otherwise, $G$ itself is a triangulation, and the assumption that $\mathcal{E}$ has no filled triangles implies that $G$ is simply a 3-vertex cycle. We will show how to form by vertex augmentation a proper supergraph $G^{\prime}$ of $G$ such that $G^{\prime}$ is a planar triangulation with no separating triangles and hence by Lemma2.1 is 4-connected.

First, we may assume that $G$ is biconnected. Otherwise, we adapt a procedure attributed to Read [37]. Consider any articulation vertex $v$, and let $u$ and $w$ be consecutive neighbors of $v$ in separate biconnected components. Add new vertex $z$ and edges $\{z, u\}$ and $\{z, w\}$. Iterating for every articulation point biconnects $G$ without adding separating triangles. Any face in the updated embedding $\mathcal{E}$ is then bounded by a simple cycle, and the following procedure is well defined.

Consider any non-triangular facial cycle $F$ in $\mathcal{E}$. Define a chord of $F$ to be a non-facial edge connecting two vertices of $F$. Consider any chord $\{x, y\}$ of $F$, and let $u$ and $v$ be the neighbors of $x$ on $F$. There can be no edge $\{u, v\}$ in $G$, for such an edge would violate planarity. Therefore embedding a new vertex $\nu(x)$ inside $F$ and adding edges $\{\nu(x), u\},\{\nu(x), x\}$, and $\{\nu(x), v\}$ cannot create a separating triangle. Let $F^{\prime}$ be the new facial cycle defined by replacing the path $(u, x, v)$ in $F$ by $(u, \nu(x), v)$, and iterate until $F^{\prime}$ has no incident chords. Then, adding a final new vertex $\nu(F)$ with edges to each vertex on $F^{\prime}$ completes the triangulation of the original face $F$ without creating separating triangles or modifying other faces. Iterating for all non-triangular facial cycles completes the process, yielding a planar triangulation $G^{\prime}$ with no additional separating triangles.

Therefore, any separating triangle $T$ in $G^{\prime}$ must have originally existed in $G$. Because $\mathcal{E}$ had no filled triangles, $T$ must have been embedded as a (triangular) face of $\mathcal{E}$. $T$ remains a face in $G^{\prime}$, however, and because $G^{\prime}$ is a triangulation, the removal of any face cannot disconnect $G^{\prime}$, thereby contradicting the existence 
of $T$.

Corollary 3.5 A graph $G$ has a rectangular layout if and only if there exists some embedding of $G$ with no filled triangles.

Proof. Thomassen [49, Thm. 2.1] proves that $G$ has a rectangular layout if and only if it is a proper subgraph of a 4-connected planar triangulation. The result then follows from Theorem 3.4 .

Biedl, Kant, and Kaufman [7] show how to transform a planar embedding without separating triangles into a 4-connected triangulation via edge augmentation, if possible. As they demonstrate, however, it is not always possible to triangulate such a graph using only edge augmentations. Furthermore, we need the vertex-augmentation method above for our algorithm in Section 4.

\subsection{Rectangle of Influence Drawings}

Finally, we link rectangular layouts to another graph visualization technique: rectangle of influence drawings. We use the definitions from Biedl, Bretscher, and Meijer [6] and Liotta et al. [27]. A (strong) closed rectangle of influence drawing of a graph $G$ is a straight-line embedding of $G$ such that the isothetic rectangular region, including the border, induced by any two vertices $u$ and $v$ contains no other vertices if and only if $\{u, v\}$ is an edge in $G$. A weak closed rectangle of influence drawing relaxes the condition so that the isothetic rectangular region, including the border, induced by any two vertices $u$ and $v$ contains no other vertices if $\{u, v\}$ is an edge in $G$. A (strong or weak) open rectangle of influence drawing is one in which all the isothetic rectangular interiors obey the respective emptyness constraints; the interiors of degenerate rectangles are defined to be those of the induced line segments. These drawings are also planar if no two edges cross.

Theorem 3.6 A graph $G$ is a contact graph of rectangles and thus admits a rectangular layout if and only if $G$ has a planar, weak, closed rectangle of influence drawing.

Proof. This follows from Corollary 3.5 and Theorem 2 of Biedl, Bretscher, and Meijer [6].

Rectangular layouts express the same class of graphs under either weak or strong adjacency constraints, but the same is not true of rectangle of influence drawings. For example, a star on three (rsp., five) leaves has a planar, weak, open (rsp., closed) rectangle of influence drawing but no strong, open (rsp., closed) rectangle of influence drawing. Liotta et al. [27] characterize graphs with strong rectangle of influence drawings. This settles an open problem raised by Biedl, Bretscher, and Meijer [6].

\section{Layouts for General Graphs}

Theorems 3.3 and 3.4 suggest an algorithm for constructing a rectangular layout for an arbitrary input graph $G$.

1. Construct an embedding $\mathcal{E}$ of $G$ with no filled triangles. If no such embedding exists, then $G$ admits no rectangular layout.

2. Vertex-augment $\mathcal{E}$ to create a proper supergraph $G^{\prime}$ of $G$ such that $G^{\prime}$ is a 4-connected triangulation.

3. Construct a rectangular dual $R$ of $G^{\prime \prime}=G^{\prime}-\{v\}$, where $v$ is any vertex added during the augmentation process in step 2 . 
4. Replace each rectangle $r$ in $R$ that corresponds to a vertex added during step 2 by a gap. The result is a rectangular layout for $G$.

Theorem 4.1 An $O\left(n^{2}\right)$-area rectangular layout can be built in $O(n)$ time for any contact graph $G$ of rectangles. If $G$ is not a contact graph of rectangles, this can be discovered in $O(n)$ time.

Proof. Biedl, Kant, and Kaufmann [7, Thm. 5.5] show how to construct an embedding $\mathcal{E}$ of $G$ with no filled triangles if one exists, or detect if no such embedding exists, both in $O(n)$ time.

The proof of Theorem 3.4 outlines a procedure to effect step 2 Finding articulation points can be done in $O(n)$ time by depth-first search [2]. Representing $\mathcal{E}$ by a standard doubly connected edge list [29] then allows all operations to be implemented in $O(n)$ time overall. In particular, after augmenting to assure biconnectivity, iterating over the faces of $\mathcal{E}$ takes $O(n)$ time plus the time to process each face. Iterating over the vertices of all the faces takes $O(n)$ time plus the time to process each vertex. Processing each vertex $x$ on each face $F$ involves checking each incident edge $e$ to see if $e$ is a chord of $F$; each such test takes $O(1)$ time, and each edge in $G$ is checked twice, once for each endpoint, for a total of $O(n)$ time. If $e$ is a chord, augmenting $F$ to replace $x$ with $\nu(x)$ also takes $O(1)$ time. Adding vertex $\nu(F)$ takes time linear in the number of vertices on $F$; over all faces this is $O(n)$ time. In all, step 2 can be done in $O(n)$ time, yielding graph $G^{\prime \prime} \supseteq G$ with $O(n)$ vertices.

Theorem 3.3 asserts that $G^{\prime \prime}$ has a rectangular dual. He [15] shows how to construct an $O\left(n^{2}\right)$-area rectangular dual of $G^{\prime \prime}$ in $O(n)$ time 3 During the construction, we simply indicate that any rectangle corresponding to a vertex in $G^{\prime \prime}-G$ should instead be rendered as a gap. Since each edge in $G^{\prime \prime}-G$ is incident to at least one vertex in $G^{\prime \prime}-G$, the result is a rectangular layout for $G$.

\subsection{General Lower Bound}

A trivial, worst-case lower bound for graphs is $\max \left\{n, \sum_{v \in V}\left\lceil\frac{\operatorname{deg}(v)}{4}\right\rceil, \sum_{v \in V}\left\lceil\frac{\operatorname{deg}(v)-2}{2}\right\rceil\right\}$, where $\operatorname{deg}(v)$ is the degree of vertex $v$. The second term comes from the fact that each vertex $v$ is represented by a rectangle, which has 4 sides; the area of that rectangle must therefore be at least $\left\lceil\frac{\operatorname{deg}(v)}{4}\right\rceil$ to accommodate all the adjacencies. This is tight in general: consider the infinite grid, in which each vertex has degree 4 and the area required is $|V|$. The third term generalizes this argument. The perimeter of $v$ 's rectangle must be at least $\operatorname{deg}(v)$ units. If the sides of the rectangle have lengths $a$ and $b$, then minimizing $a b$ subject to $a+b \geq d / 2$ yields that $a b \geq\left\lceil\frac{\operatorname{deg}(v)-2}{2}\right\rceil$.

To show a worst-case lower bound that matches our upper bound, first define an $n$-rung ladder to be a graph on at least $n+2$ vertices- $L, R$, and $x_{i}$ for $1 \leq i \leq n$-with edges $\left\{L, x_{i}\right\}$ and $\left\{x_{i}, R\right\}$ for $1 \leq i \leq n$ and paths (possibly including additional vertices) connecting $x_{i}$ to $x_{i+1}$ for $1 \leq i<n$. We call the $x_{i}$ 's the rungs and $L$ and $R$ the struts of the ladder.

In a rectangular layout, call some rectangle $r$ above some rectangle $s$ if the lowest extent of $r$ is no lower than the highest extent of $s$. Symmetrically define below, right of, and left of. Call a set of rectangles vertically (rsp., horizontally) stacked if their above (rsp., left of) relationships form a total order. A set of rectangles is vertically (rsp., horizontally) aligned if they have pairwise identical projections onto the $x$-axis (rsp., $y$-axis). We use the length of a rectangle to mean the maximum of its width and height.

Lemma 4.2 Assume $n \geq 3$. Any rectangular layout for an $n$-rung ladder must possess one of the following sets of properties:

\footnotetext{
${ }^{3}$ He does not explicitly state the area of the dual resulting from his construction, but the bound is easily derived.
} 

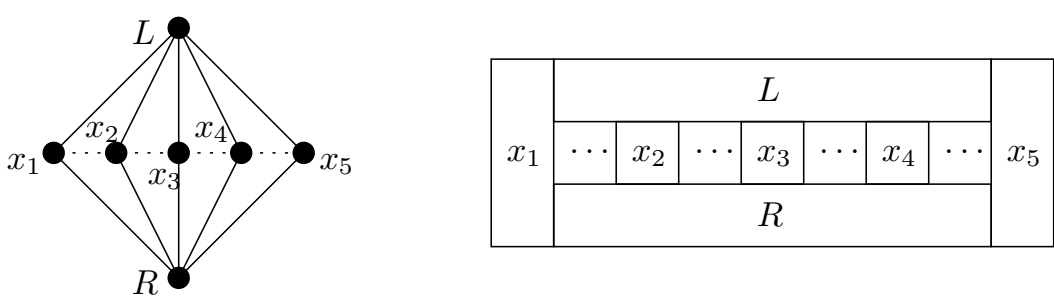

Figure 3: A 5-rung ladder (dotted lines indicate paths) and a possible layout. In any layout, all the $x_{i}$ must be stacked, and the rectangles $x_{2}, x_{3}$, and $x_{4}$ must be aligned as shown between $L$ and $R$. The only other variations also align rectangle(s) $x_{1}$ and/or $x_{5}$, but the width remains at least 5 .
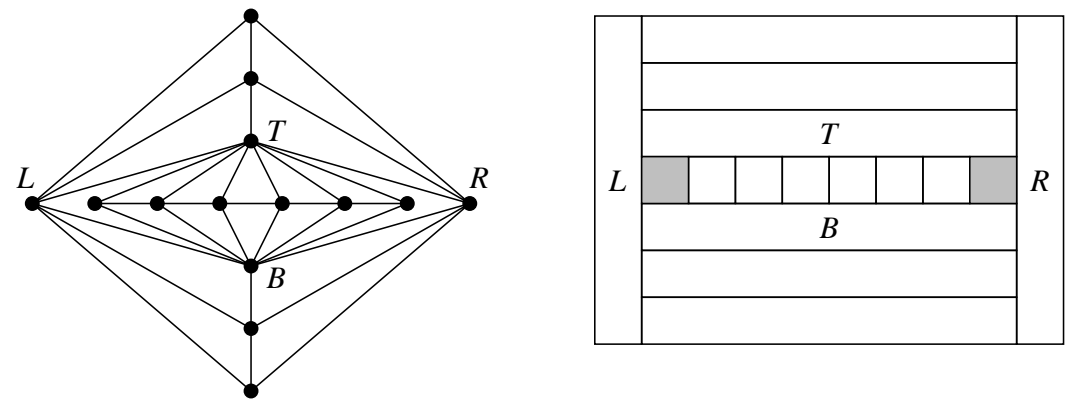

Figure 4: A (6,6)-ladder and a possible layout.

1. width at least $n$ and height at least 3 ; rectangles $x_{i}$ for $1 \leq i \leq n$ all horizontally stacked; and rectangles $x_{i}$ for $1<i<n$ all horizontally aligned between $L$ and $R$; or

2. height at least $n$ and width at least 3 ; rectangles $x_{i}$ for $1 \leq i \leq n$ all vertically stacked; and rectangles $x_{i}$ for $1<i<n$ all vertically aligned between $L$ and $R$.

Proof. Consider the path $\left(x_{1}, S_{1}, x_{2}, S_{2}, \ldots, x_{n-1}, S_{n-1}, x_{n}\right)$, where the $S_{i}$ 's are possibly null paths, connecting the $x_{i}$ 's. We prove the lemma by induction on the total number of vertices in the $S_{i}$ 's. Refer to Figure 3 . We interchange the notion of vertices and rectangles and rely on context to disambiguate.

The base case is when all the $S_{i}$ 's are null; i.e., there is a direct path $\left(x_{1}, x_{2}, \ldots, x_{n}\right)$. Let $L$ be placed above $R$; other cases are symmetric. Only $x_{1}$ and $x_{n}$ may be to the sides (left and right) of $L$ and $R$, for if a different $x_{i}$ were, say, to the left of $L$ and $R$, abutting both, then one of $x_{i-1}$ and $x_{i+1}$ would not be able to abut both $L$ and $R$. Thus, $x_{2}, \ldots, x_{n-1}$ must be below $L$ and above $R$; that they must each abut both $L$ and $R$ therefore implies that these rectangles must be horizontally aligned. If $x_{1}$ and/or $x_{n}$ are to the sides of $L$ and $R$, all the $x_{i}$ 's are stacked; if $x_{1}$ and $x_{n}$ are also below $L$ and above $R$, all the rectangles are horizontally aligned and hence also horizontally stacked. That the rectangles are horizontally stacked implies that the width is $n$. The height follows by construction. This proves the base case.

Given a layout for any graph, removing the rectangle corresponding to some vertex-i.e., turning it into a gap-must produce a layout for the corresponding proper subgraph. This proves the inductive step.

Define an $(i, j)$-ladder to be a graph on $i+j+2$ vertices: an $i$-rung (external) ladder defined by some vertices $L, R$, and $x_{k}$ for $1 \leq k \leq i$, united with a $j$-rung (internal) ladder defined by $x_{\lfloor i / 2\rfloor}, x_{\lfloor i / 2\rfloor+1}$, and $y_{k}$ for $1 \leq k \leq j$. See Figure 4 
Theorem 4.3 For $n \geq 4$, any layout for an $(n, n)$-ladder has area $\Omega\left(n^{2}\right)$.

Proof. Lemma 4.2 applied to the external ladder shows that the height (or, rsp., width) of any layout is at least $n$ and that the rectangles $x_{\lfloor n / 2\rfloor}$ and $x_{\lfloor n / 2\rfloor+1}$ are vertically (or, rsp., horizontally) aligned between $L$ and $R$. Say the height is $n$; the other case is symmetric. Then Lemma 4.2 shows that the width induced by the internal ladder is at least $n$. The theorem follows.

\section{Layouts for Trees}

We present an algorithm that constructs $O(n \log n)$-area rectangular layouts for trees. We then show a matching worst-case lower bound. There do exist trees with better layouts, however, and we constructively show an infinite class of trees that have $O(n)$-area layouts. As with general graphs, this leaves open the problem of devising better approximation algorithms for trees.

\subsection{General Algorithm}

Given an undirected tree, $T=(V, E)$, assume $T$ is rooted at some vertex $r$; if not, pick an arbitrary root. A simple Algorithm A lays out $T$ as follows. For all $v \in V$, let $\operatorname{desc}(v)$ be the number of descendants of $v$. Each vertex $v$ is represented as a rectangle of height 1 and width $\operatorname{desc}(v)$. For any $v$, the rectangles for its children are placed under the rectangle for $v$. The rectangle for the root appears on top. For simplicity, we allow corners to meet trivially; with our construction, we can eliminate this problem at a constant-factor area penalty. Similarly, we ignore the issue of strong versus weak layouts. See Figure 5 a).

Lemma 5.1 Algorithm A produces a layout of $T$ of area $n \cdot \operatorname{depth}(T)$.

Proof. The assertion about area is straightforward. Correctness follows by induction from the fact that each rectangle is wide enough to touch all the rectangles for its vertex's children plus one unit for itself.

Consider a partition of $T$ into a collection of vertex-disjoint paths. Algorithm A generalizes into Algorithm $\mathrm{B}$ by abutting all rectangles in a single path horizontally and abutting rectangles for deeper children in the partition vertically. Details follow. Refer to Figure 5 (b).

For a path $P=\left(u_{0}, \ldots, u_{k}\right)$, in top-down order, define $t(P)=u_{0}$, i.e., the vertex in $P$ of maximum height. Define $\operatorname{desc}(P)=\operatorname{desc}(t(P))$. Define $s\left(u_{k}\right)=\operatorname{desc}\left(u_{k}\right)$, and for $0 \leq i<k$, define $s\left(u_{i}\right)=$ $\operatorname{desc}\left(u_{i}\right)-\operatorname{desc}\left(u_{i+1}\right)$. Note that $\sum_{x \in P} s(x)=\operatorname{desc}(P)$.

Each path $P=\left(u_{0}, \ldots, u_{k}\right)$ is represented as a rectangle of height 1 and width $\operatorname{desc}(P)$, which is partitioned into rectangles of width $s\left(u_{0}\right), \ldots, s\left(u_{k}\right)$, each representing the corresponding $u_{i} \in P$. The rectangle for path $P$ with $t(P)=r$ is placed on top. Under the rectangle for each $u_{i} \in P$ (for each $P$ ) are placed the rectangles for each path $P^{\prime}$ such that $t\left(P^{\prime}\right)$ is a child of $u_{i}$.

Lemma 5.2 Algorithm B produces a layout of $T$.

Proof. Consider any vertex $x$. We need only account for the adjacency $(x, p(x))$ (assuming $x \neq r$ ), because any child $u$ of $x$ is accounted for by the adjacency $(u, p(u))$.

Denote by $B(x)$ the rectangle representing $x$. If $x \neq r$, then $B(x)$ overlaps $B(p(x))$, for either $x$ and $p(x)$ are in a common path, in which case their rectangles share a vertical side, or else $B(x)$ is layed out underneath $B(p(x))$. The construction assures that $B(p(x))$ is wide enough in this latter case.

Consider the compressed tree $C(T)$, formed by compressing each path $P$ into a super-vertex, with edges to each super-vertex $P^{\prime}$ such that $t\left(P^{\prime}\right)$ is a child of some $x \in P$. 

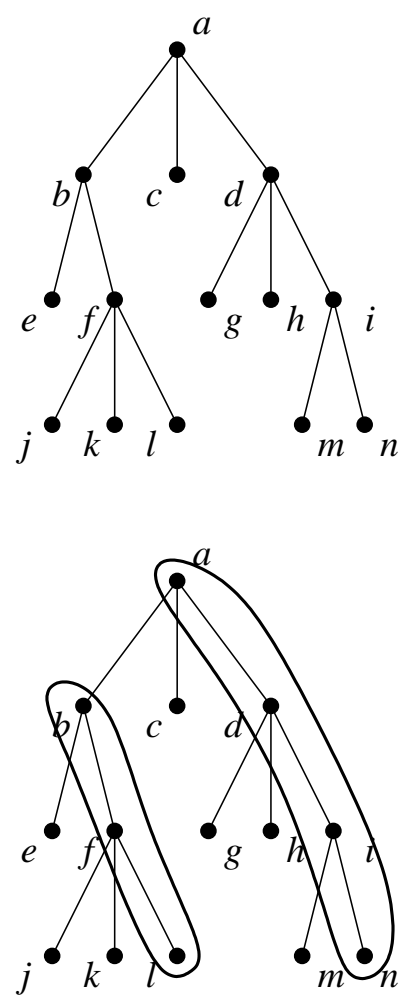

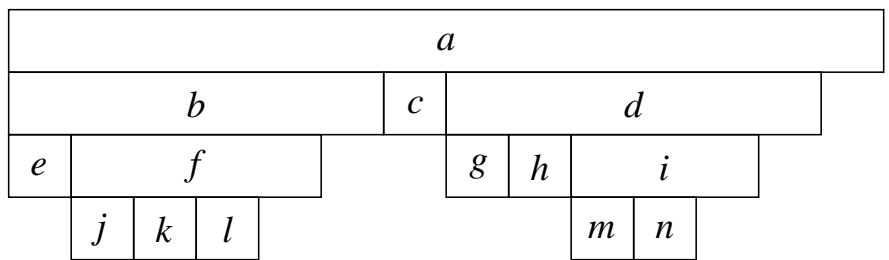

(a)

\begin{tabular}{|l|l|l|l|l|l|l|l|l|l|l|l|}
\hline \multicolumn{7}{|c|}{$a$} & \multicolumn{2}{|c|}{$d$} & \multicolumn{2}{|c|}{$i$} & $n$ \\
\hline \multicolumn{2}{|c|}{$b$} & \multicolumn{2}{|c|}{$f$} & $l$ & $c$ & & $g$ & $h$ & & & \\
$y$ & & & &
\end{tabular}

Figure 5: (a) A tree and the result of applying Algorithm A. The width of each rectangle is the number of descendants of the corresponding vertex; e.g., the width of $f$ is 4 , and the width of $d$ is 6 . (b) A partition of the tree into paths - uncircled nodes form singleton paths - and the application of Algorithm B to the partition.

Lemma 5.3 Algorithm B produces a layout of $T$ of area $n \cdot \operatorname{depth}(C(T))$.

Proof. Algorithm B produces a one-unit high collection of rectangles for each distinct depth in $C(T)$. Each such collection is of width no more than $n$ (the total number of descendants of the paths at that depth).

We use the heavy-path partition of $T$, as defined by Harel and Tarjan [14] and later used by Gabow [11] 4 Call tree edge $(v, p(v))$ light if $2 \cdot \operatorname{desc}(v) \leq \operatorname{desc}(p(v))$, and heavy otherwise. Since a heavy edge must carry more than half the descendants of a vertex, each vertex can have at most one heavy edge to a child, and therefore deletion of the light edges produces a collection of vertex-disjoint heavy paths. (A vertex with no incident heavy edges becomes a singleton, called a trivial heavy path.)

Theorem 5.4 Algorithm B applied to the heavy-path partition of T produces a layout of area $O(n \log n)$ in $O(n)$ time.

Proof. The compressed tree, $C(T)$, is constructed by contracting each heavy path in $T$ into a single supervertex. Each tree edge in $C(T)$ corresponds to a light edge of $T$. Since there are $O(\log n)$ light edges on the

\footnotetext{
${ }^{4}$ Tarjan [47] originally introduced heavy-path partitions, but defined in different terms; Schieber and Vishkin [40] later used yet another variant.
} 


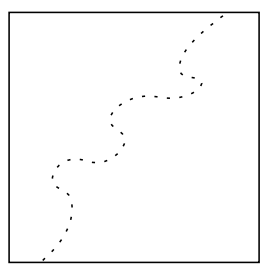

(a)

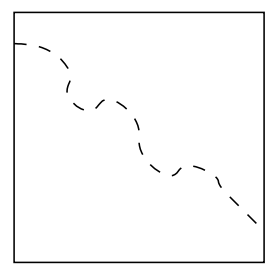

(b)

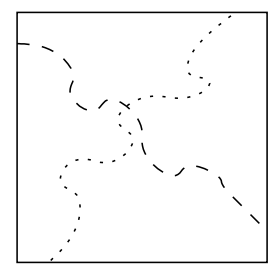

(c)

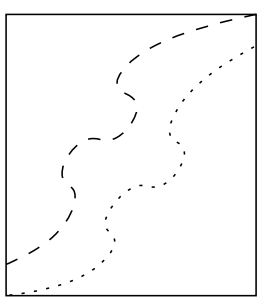

(d)

Figure 6: Bounding boxes and extremal paths for various layouts. (a) Layout $L_{1}$ for $G_{1}$; (b) Layout $L_{2}$ for $G_{2}$; each of size $d$-by- $d$. (c) Impossible $d$-by- $d$ layout for $G$ containing $G_{1}$ and $G_{2}$ as subgraphs; any corresponding extremal paths would have to cross. (d) $d$-by- $(d+1)$ layout for $G$. The extremal path for $L_{1}$ is dotted, and that for $L_{2}$ is dashed. By extending $G$ in one dimension, the extremal paths need not cross.

path from any vertex to the root of $T, C(T)$ has depth $O(\log n)$. The area bound follows from Lemmas 5.2 and 5.3. $C(T)$ can be built in $O(n)$ time after a depth-first search; the rest of the algorithm performs $O(1)$ work per vertex.

\subsection{General Lower Bound}

We show there exists an infinite family of trees that require $\Omega(n \log n)$ area for any layout. First we show that any layout of a binary tree has $\Omega(\log n)$ length in each dimension.

We define the notion of paths in layouts. A path in layout $L$ is a sequence $\left(r_{1}, \ldots, r_{\ell}\right)$ of rectangles in $L$ such that for each $1 \leq i<\ell$, the boundaries of $r_{i}$ and $r_{i+1}$ overlap. A path in a strong layout thus corresponds to a path in the underlying graph. A vertical extremal path of $L$ is a path that touches both the top and bottom of $L$ 's bounding box $B$; similarly, define a horizontal extremal path to touch the left and right sides of $B$. An extremal path that touches opposite corners of $B$ is both vertical and horizontal. By definition, every layout has at least one vertical and at least one horizontal extremal path, possibly identical.

Consider graph $G$ with some layout $L$ and some subgraph $G^{\prime}$. $L$ contains a sub-layout $L^{\prime}$ for $G^{\prime}$. Any extremal path in $L^{\prime}$ induces a path in $L$. Two sub-layouts are disjoint if their induced subgraphs are disjoint. Extremal paths for disjoint sub-layouts may not cross in $L$, for this would imply two non-disjoint rectangles, a fact codified as follows.

Fact 5.5 Consider graph $G$, some layout $L$ of $G$, and any two disjoint, connected subgraphs $G_{1}$ and $G_{2}$ of G. Extremal paths for the corresponding sub-layouts $L_{1}$ and $L_{2}$ may not cross in $L$.

Lemma 5.6 Let $G_{1}$ and $G_{2}$ have minimal area layouts with length at least $d$ in each dimension. Let $G$ contain both $G_{1}$ and $G_{2}$ as subgraphs. Then any layout $L$ of $G$ has length at least $d+1$ in at least one dimension.

Proof. Since $L$ contains sub-layouts $L_{1}$ and $L_{2}$ for $G_{1}$ and $G_{2}$, rsp., by assumption $L$ has length at least $d$ in both dimensions. If $L$ has width and height both $d$, then any horizontal extremal path for $L_{1}$ must cross any vertical extremal path for $L_{2}$, contradicting Fact 5.5 Thus, $L$ must have width or height at least $d+1$. (See Figure 6.)

Lemma 5.7 Let $G_{1}$ and $G_{2}$ be graphs such that all their layouts have length at least $d$ in each dimension. Let $G$ be formed by adding a new vertex $r$, adjacent to one vertex in each of $G_{1}$ and $G_{2}$. Then in any layout 


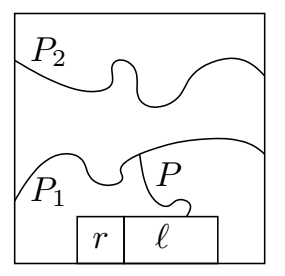

(a)

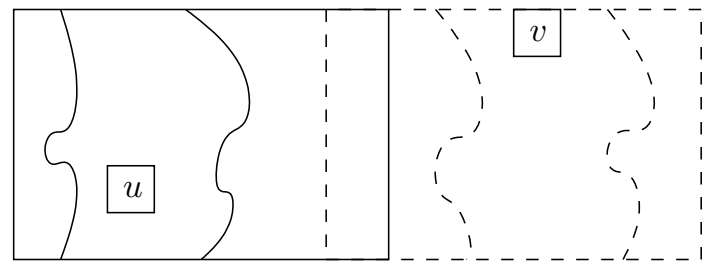

(b)

Figure 7: (a) A bounding box of width $d$ containing a layout $L$ that includes two sub-layouts $L_{1}$ and $L_{2}$, each of minimum dimension $d$. If $r$ is adjacent to the bottom of $L$, let $\ell$ be a rectangle in $L_{1}$ adjacent to $r$. There is some path $P$ in $L_{1}$ connecting $\ell$ to a horizontal extremal path $P_{1}$ of $L_{1}$ and $L$. No horizontal extremal path of $L_{2}$ can intersect the closed curves formed by $P, P_{1}$, and the bounding box of $L$. Thus $P_{2}$ is above $P_{1}$, but then $r$ cannot be connected to $P_{2}$ without crossing $P_{1}$. (b) Bounding boxes for layouts of two $T_{i-1}$ 's, rooted at $u$ (solid) and $v$ (dashed). If both layouts are of height $\left\lfloor\frac{i-2}{2}\right\rfloor$, then extremal paths (dashed for the $T_{i-1}$ rooted at $v$ ) separate $u$ from $v$, and $r$ cannot be placed within this height.

$L$ of $G$ with length $d$ in some dimension, $r$ cannot be incident to a length-d side of the bounding box of $L$. Furthermore there exist extremal paths in the length-d dimension to either side of $r$.

Proof. (Refer to Figure 7a).) Assume to the contrary that $L$ has width $d$ and $r$ is adjacent to the bottom (sym., top) of $L$; the argument for height $d$ is symmetric. $L$ induces layouts $L_{1}$ of $G_{1}$ and $L_{2}$ of $G_{2}$, each by assumption with length at least $d$ in each dimension $d$. Let $r$ be adjacent to a rectangle $\ell$ of $L_{1}$ (sym., $L_{2}$ ). Then there must be a path $P$ from $\ell$ that intersects a horizontal extremal path $P_{1}$ of both $L_{1}$ and $L$. The union of $P$ and one side of this extremal path forms a closed curve with the bounding box that contains $r$.

Now consider a horizontal extremal path $P_{2}$ of $L_{2}$, which is also a horizontal extremal path of $L$. There must be a path $P^{\prime}$ in $L_{2}$ connecting $P_{2}$ to $r . P_{2}$ cannot intersect the closed curve defined above without creating non-disjoint rectangles; thus $P_{2}$ is above $P_{1}$. But then by the Jordan curve theorem [30, Section 8-13], $P^{\prime}$ itself must cross the curve to reach $P_{2}$, which again would create non-disjoint rectangles.

If $r$ is above $P_{2}$, a similar contradiction holds. Thus, $r$ must be between $P_{1}$ and $P_{2}$.

Define $T_{i}$ to be a complete binary tree on $2^{i}$ leaves.

Lemma 5.8 Any layout for $T_{i}$ has length at least $\lfloor i / 2\rfloor$ in each dimension.

Proof. The theorem is true for $i=0$ and $i=1$. Assuming it is true up to $i-1$, we prove it by induction for $i \geq 2$. Denote by $r$ the root of $T_{i}$ and by $u$ and $v$ the roots of the $T_{i-1}$ 's rooted at the children of $r$. By induction, each $T_{i-2}$ rooted at children of $u$ and $v$ has length at least $\left\lfloor\frac{i-2}{2}\right\rfloor$ in each dimension. By Lemma 5.6 therefore, the layouts for the $T_{i-1}$ subtrees rooted at $u$ and $v$ each have at least one dimension of length $\left\lfloor\frac{i-2}{2}\right\rfloor+1=\lfloor i / 2\rfloor$. If either sub-layout has both dimensions this large, we are done. If one sub-layout has width $\lfloor i / 2\rfloor$ and the other height $\lfloor i / 2\rfloor$, we are similarly done.

Therefore, assume the sub-layouts for both $T_{i-1}$ 's have height $\left\lfloor\frac{i-2}{2}\right\rfloor$. (Symmetrically argue if both widths are this small.) Also assume that in the layout for $T_{i}, r$ is not placed on top of the two sub-layouts, or the height grows by the required one unit. By Lemma 5.7 neither $u$ nor $v$ may be adjacent to the left or right side of their layouts, and furthermore, there are vertical extremal paths due to their own children that separate $u$ from $v$. But then by Jordan curve arguments, there cannot be paths connecting both $u$ and $v$ to $r$ without violating rectangle disjointedness. (See Figure 7b).) 


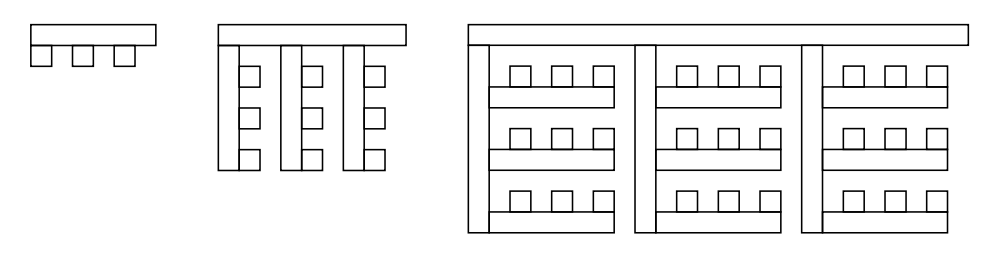

Figure 8: Layouts $L_{3}^{3}$ (left), $L_{9}^{3}$ (middle), and $L_{27}^{3}$ (right).

Assume $n=2^{k}$ for some integer $k \geq 1$. Let $S_{n}$ be a tree formed by linking the root of $T_{k}$ to the root of a star with $n$ leaves.

Theorem 5.9 Any layout $L_{n}$ for $S_{n}$ has $\Omega(n \log n)$ area.

Proof. $L_{n}$ includes sub-layouts for $T_{k}$ and the star on $n$ leaves. Lemma 5.8 implies that the length of each dimension of $L_{n}$ is $\Omega(\log n)$. The only layout for a star with $n$ leaves is a rectangle for the root with $n$ rectangles around its perimeter. Thus, at least one dimension of the star layout is of length $\Omega(n)$. The theorem follows.

\subsection{Linear Area for Complete Trees}

Let $T_{n}^{k}$ be a complete tree of arity $k$ on $n$ leaves and $L_{n}^{k}$ a corresponding strong layout. $L_{1}^{k}$ is the unit square. For higher $n$, construct $L_{n}^{k}$ as follows. Recursively construct the $k L_{n / k}^{k}$ sub-layouts, assuming the root of each one has unit length in one dimension. Attach the roots of the sub-layouts by their unit-length sides to a root rectangle of height 1 and appropriate width, leaving one unit of horizontal space to the right of each sub-layout. See Figure 8

Theorem $5.10 L_{n}^{k}$ is of area $O(n)$.

Proof. For clarity, we drop the superscripts. Denote by $h_{n}$ the height and $w_{n}$ the width of $L_{n}$. Then $h_{1}=w_{1}=1 ; h_{k}=2, w_{k}=2 k$; and for higher $n$, we have the following type-1 recurrence:

$$
\begin{aligned}
& h_{n}=1+w_{n / k} ; \\
& w_{n}=k h_{n / k}+k .
\end{aligned}
$$

Simplifying:

$$
\begin{aligned}
& h_{n}=k h_{n / k^{2}}+k+1 ; \\
& w_{n}=k w_{n / k^{2}}+2 k .
\end{aligned}
$$

Solving these recurrences for $n=k^{c}$ yields

$$
h_{n}=\Theta\left(k^{\lfloor c / 2\rfloor}\right) ; w_{n}=\Theta\left(k^{\lceil c / 2\rceil}\right) .
$$

Therefore, the area, which is $h_{n} w_{n}$, is $\Theta(n)$.

An alternative layout yields a family of layouts of linear area, but with elastic widths and heights. Rather than attaching the roots of the sub-layouts to the root of the layout by their unit-length sides, consider attaching them by their other sides. This establishes a recurrence of the form

$$
\begin{aligned}
& h_{n}=1+h_{n / k} ; \\
& w_{n}=k w_{n / k}+k ;
\end{aligned}
$$


which yields the solution $h_{n}=\Theta\left(\log _{k} n\right), w_{n}=\Theta(n)$. We call this a type-2 recurrence.

Combining the above two recurrences, we can prove the following.

Theorem 5.11 For any constant $\alpha \in\left[\frac{1}{2}, 1\right)$, a layout for $T_{n}^{k}$ exists with width $\Theta\left(n^{\alpha}\right)$ and height $\Theta\left(n^{1-\alpha}\right)$.

Proof. If we apply a type- 1 recurrence $2 m$ times, we obtain the recurrence

$$
\begin{aligned}
& h_{n}=(1+k)\left(1+k+\cdots+k^{m-1}\right)+k^{m} h_{n / k^{2 m}} \\
& w_{n}=2 k\left(1+k+\cdots+k^{m-1}\right)+k^{m} w_{n / k^{2 m}}
\end{aligned}
$$

which simplified yields

$$
\begin{aligned}
& h_{n}=\Theta\left(k^{m}\right)+k^{m} h_{n / k^{2 m}} ; \\
& w_{n}=\Theta\left(k^{m}\right)+k^{m} w_{n / k^{2 m}} .
\end{aligned}
$$

Similarly, a type- 2 recurrence applied $2 \ell$ times yields

$$
\begin{aligned}
& h_{n}=2 \ell+h_{n / k^{2 \ell}} ; \\
& w_{n}=k^{2 \ell}+k^{2 \ell} w_{n / k^{2 \ell}} .
\end{aligned}
$$

Consider a layout in which we apply a type- 1 construction $2 m$ times and then a type- 2 construction $2 \ell$ times and then repeat. The recurrence governing this construction is given by a combination of the above two recurrences:

$$
\begin{aligned}
& h_{n}=\Theta\left(k^{m}\right)+k^{m}\left(2 \ell+h_{n / k^{(2 \ell+2 m)}}\right) \\
& w_{n}=\Theta\left(k^{m}\right)+k^{m}\left(k^{2 \ell}+k^{2 \ell} w_{n / k^{(2 \ell+2 m)}}\right)
\end{aligned}
$$

which simplifies to

$$
\begin{aligned}
& h_{n}=\Theta\left(k^{m} \ell\right)+k^{m} h_{n / k^{(2 \ell+2 m)}} ; \\
& w_{n}=\Theta\left(k^{m+2 \ell}\right)+k^{m+2 \ell} w_{n / k^{(2 \ell+2 m)}} .
\end{aligned}
$$

Solving these recurrences and setting $\alpha=1-m /(2 m+2 \ell)$, we get $h_{n}=\Theta\left(n^{1-\alpha}(1+\ell)\right)$ and $w_{n}=\Theta\left(n^{\alpha}\right)$. For any $1 / 2 \leq \alpha<1$ we can find constants $\ell$ and $m$ that satisfy this equation.

\section{Area Optimality}

\subsection{NP-Hardness of Generating Optimal Layouts}

Recall the problem of numerical matching with target sums [13]. Given are disjoint sets $X$ and $Y$, each of $m$ elements, a size $s(a) \in \mathbb{Z}^{+}$for each $a \in X \cup Y$, and a target vector $B=\left(B_{1}, \ldots, B_{m}\right)$ with each $B_{i} \in \mathbb{Z}^{+}$. The problem is to determine if $X \cup Y$ can be partitioned into $m$ disjoint sets $A_{1}, \ldots, A_{m}$, each containing exactly one element from each of $X$ and $Y$, such that for $1 \leq i \leq m, \sum_{a \in A_{i}} s(a)=B_{i}$. The problem is strongly NP-hard in general. Consider some instance $\mathcal{I}$ of numerical matching with target sums. Assume without loss of generality that

$$
\sum_{x \in X} s(x)+\sum_{y \in Y} s(y)=\sum_{i=1}^{m} B_{i},
$$




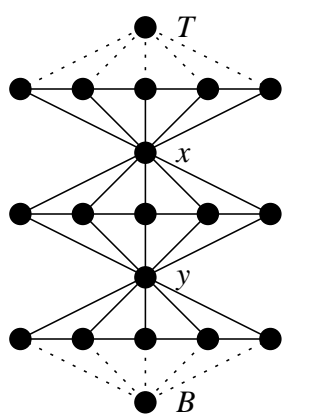

(a)

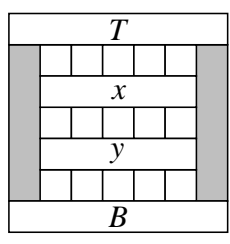

(b)

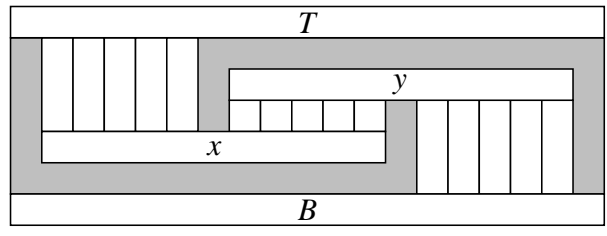

(c)

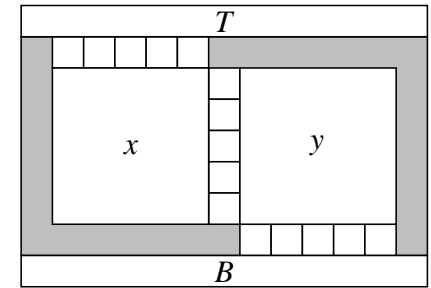

(d)

Figure 9: (a) A 5-accordion enclosed by $T$ and $B$. The accordion edges are solid; the enclosing edges are dotted. (b)-(d) Layouts with (b) $y$ below $x$; (c) $x$ below $y$; and (d) neither $x$ nor $y$ below the other.

or else $\mathcal{I}$ has no solution. We will construct a graph $G(\mathcal{I})$ that has an optimal layout of certain dimensions if and only if $\mathcal{I}$ has a solution.

Define an $n$-accordion to be a graph on $3 n+2$ vertices: three disjoint, simple paths of length $n$ each; two additional vertices $x$ and $y$; an edge between $x$ and each vertex on the first and second paths; and an edge between $y$ and each vertex on the second and third paths. An enclosed accordion is an accordion augmented (enclosed) by two additional vertices $T$ and $B: T$ adjacent to each vertex on the first path, and $B$ adjacent to each vertex on the third path. See Figure 9 (a).

Lemma 6.1 Assume $n \geq 3$. In any layout of an enclosed $n$-accordion such that $T$ appears above (sym., below) $B$ and no rectangle corresponding to an accordion vertex is left or right of $T$ or $B$, the accordion rectangles form a bounding box of height at least 5 and width at least $n$; furthermore, to achieve height 5 and width $n$ simultaneously, the accordion rectangles must form a dissection. Symmetrically, in any layout of an enclosed $n$-accordion such that $T$ appears left (sym., right) of $B$ and no rectangle corresponding to an accordion vertex is above or below $T$ or $B$, the accordion rectangles form a bounding box of height at least $n$ and width at least 5; furthermore, to achieve height $n$ and width 5 simultaneously, the accordion rectangles must form a dissection.

Proof. We prove the case in which $T$ is above $B$; the other cases are symmetric. Refer to Figure 9 The width lower bound follows, because all vertices on the first path must be adjacent to $T$ 's bottom boundary.

By assumption, in any layout $x$ must be below $T$ and $y$ above $B$. Lemma 4.2 implies that the width or height of the ladder between $x$ and $y$ must be at least $n$. If the height is at least $n$ (as in Figure 9d)), the height lower bound follows from the mutual non-adjacency of $x, y, T$, and $B$ and the lower bound on $n$. If the height of the $x-y$ ladder is less than $n$, then Lemma 4.2 implies that the width must be at least $n$ and the height at least 3. Because neither $x$ nor $y$ can abut $T$ or $B$, there must be at least one additional unit of height each above and below the $x-y$ ladder to connect it $T$ and $B$ via the intermediate vertices. Example configurations are depicted in Figures 9 (b)-(d). Thus the overall height of the accordion must be at least 5 .

To achieve height 5 and width $n$ simultaneously, the $x-y$ ladder itself must be of height 3 , by the same argument that 2 additional units of height are required to connect it to $T$ and $B$. By Lemma 4.2 , therefore, the $x-y$ ladder must have width at least $n$, with the rectangles other than $x$ and $y$ stacked and the middle ones aligned horizontally. If they were simply stacked, however, then the width of $x$ and $y$ would be only 3 , which would not suffice to place the rectangles between them and $T$ and $B$. Hence all of the rectangles of the $x$-y ladder other than $x$ and $y$ must be aligned, which then implies that the rectangles between $x$ 
and $T$ and those between $y$ and $B$ must also be aligned, as shown in Figure 9 b), to meet the overall width assumption. This forms a dissection, as claimed.

Let $x_{1}, \ldots, x_{m}$ (rsp., $\left.y_{1}, \ldots, y_{m}\right)$ denote the sizes of the elements of $X$ (rsp., $Y$ ) in $\mathcal{I}$; define $B^{*}=$ $\max \left\{B_{i}: 1 \leq i \leq m\right\}$; and define $\Delta_{i}=2 B^{*}-B_{i} \geq 1$ for $1 \leq i \leq m$. Graph $G(\mathcal{I})$ is formed from the following components.

- vertices $X, Y, t, b, g^{*}$, and $g_{i}$ for $0 \leq i \leq m$;

- an $x_{i}$-rung ladder $R_{i}$ and a $y_{i}$-rung ladder $S_{i}$ for $1 \leq i \leq m$;

- a $\Delta_{i}$-accordion, denoted $\mathcal{A}_{i}$, for $1 \leq i \leq m$;

- a $\left(2 B^{*}+2\right)$-accordion, denoted $\mathcal{A}^{*}$.

The components are arranged as follows. (See Figure 10)

- $X$ and $Y$ are each adjacent to $t, b, g^{*}$ and $g_{i}$ for $0 \leq i \leq m$;

- $t$ is adjacent to $g_{0}$ and $b$ to $g^{*}$;

- $g_{i-1}$ and $g_{i}$ enclose $\mathcal{A}_{i}$ for $1 \leq i \leq m$;

- $g_{m}$ and $g^{*}$ enclose $\mathcal{A}^{*}$;

- $X$ is adjacent to the struts of $R_{i}$ for $1 \leq i \leq m$;

- $Y$ is adjacent to the struts of $S_{i}$ for $1 \leq i \leq m$.

Lemma 6.2 Let $\mathcal{I}$ be an instance of numerical matching with target sums. $\mathcal{I}$ has a solution if and only if $G(\mathcal{I})$ has a rectangular layout of width no more than $2 B^{*}+6$ and height no more than $6 m+9$ (or vice versa).

Proof. The subgraph of $G(\mathcal{I})$ induced by $X, Y, t, b, g^{*}$, the $g_{i}$ 's, and paths through the accordions connecting $t, g_{0}$, etc. through $b$, create a global $(m+4)$-rung ladder. Scaling if necessary, we can assume all $\Delta_{i} \geq 3$. Lemmas 4.2 and 6.1 together imply that any layout for $G(\mathcal{I})$ respects the following (up to width/height symmetry). (Refer to Figure 11)

1. The overall height is at least $6 m+9$, because the $m+1$ enclosed accordions, each accordion itself of height 5 , are all aligned, and the $m+4$ rungs of the global ladder are stacked.

2. The overall width is at least $2 B^{*}+6: 2 B^{*}+2$ for $\mathcal{A}^{*}, 2$ to separate it from $X$ and $Y$, and 1 each for $X$ and $Y$.

(11) is true regardless of whether rectangle $t$ (rsp., b) is above (rsp., below) $X$ and $Y$ or in between. That is, the subgraph induces an incompressible sub-layout: these components must have the indicated heights and/or widths in any layout.

Refer to the sub-layout including $g_{i-1}, \mathcal{A}_{i}$, and $g_{i}$ as layer $i$. Accordion $\mathcal{A}_{i}$ creates gap(s) of total width $w(i)=2 B^{*}+4-\Delta_{i}$ between $X$ and $Y$ at layer $i$. The idea is to fill each such gap with a sub-layout for some ladder adjacent to $X$ or $Y$. Each such ladder sub-layout must consist of two struts, attached to $X$ (or $Y$ ), with the rungs between them (except for possibly one of them) but separated from $X$ and $Y$. See Figure 


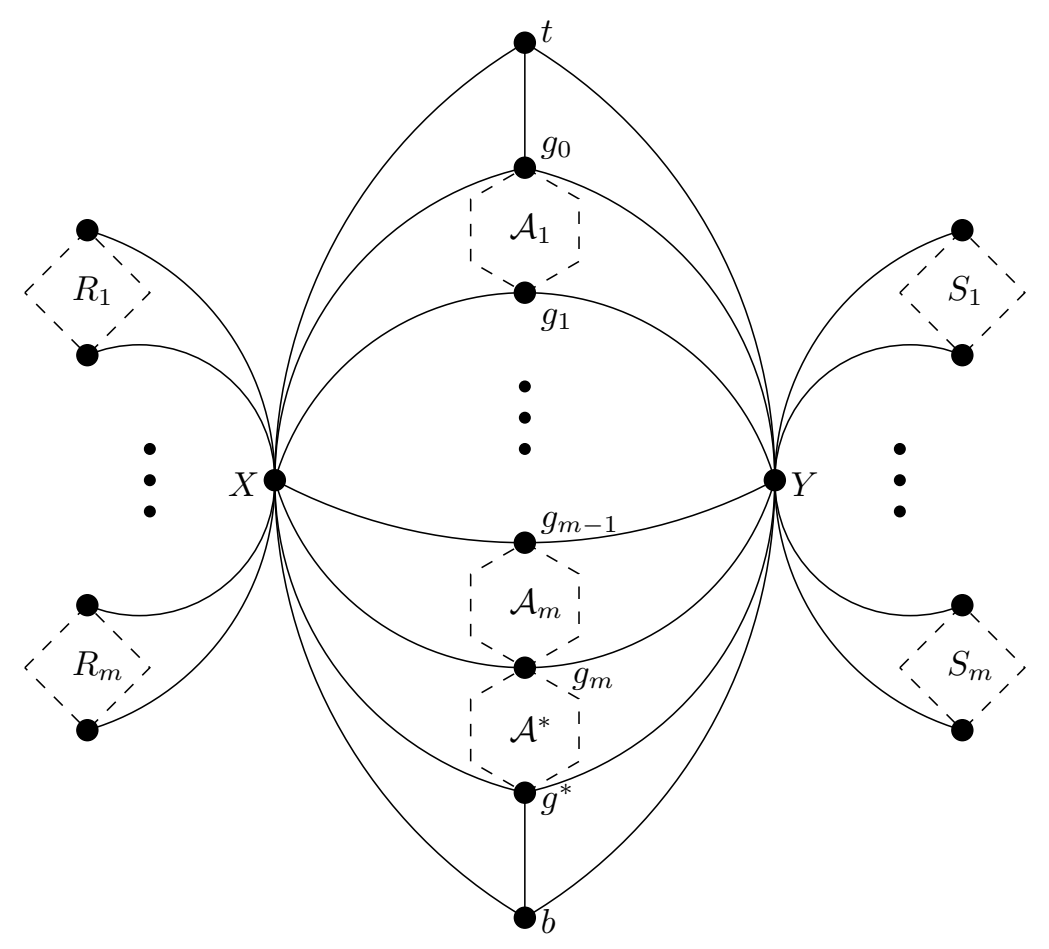

Figure 10: The graph $G(\mathcal{I})$ for some instance $\mathcal{I}$ of numerical matching with target sums. The accordions are denoted by dashed hexagons and the ladders by dashed diamonds.

11 b). Ladder $R_{i}$ has $x_{i}$ rungs, and its sub-layout must therefore have length at least $x_{i}+1$ ( $x_{i}$ for rungs plus one unit to separate the rungs from $X$ ). Similarly, ladder $S_{i}$ 's sub-layout must have length at least $y_{i}+1$.

Assume $\mathcal{I}$ has a solution. Re-index the elements of $X$ and $Y$ so that $x_{i}+y_{i}=B_{i}$ for $1 \leq i \leq m$. Then ladders $R_{i}$ and $S_{i}$ fit in the layer- $i$ gaps without increasing the width of each layer between $X$ and $Y$ from the minimum $2 B^{*}+4$. To see this, note that the width required for the sub-layouts at layer $i$ is $x_{i}+y_{i}+\Delta_{i}+4=2 B^{*}+4: x_{i}+1$ for $R_{i}, y_{i}+1$ for $S_{i}, \Delta_{i}$ for $\mathcal{A}_{i}$, and 2 units to separate $\mathcal{A}_{i}$ from $R_{i}$ and $S_{i}$. Also, each ladder sub-layout fits within the height-5 lower bound of layer $i$. Therefore, the width remains $2 B^{*}+6$, the height remains $6 m+9$, and so the desired layout exists.

Assume $G(\mathcal{I})$ has a layout of the hypothesized dimensions. The incompressibility argument above implies that each ladder must fit into a gap in one of the layers. No two ladders may be placed in the same gap, for the height of the layer would have to grow, violating the height assumption. Re-index the elements of $X$ and $Y$ so that for $1 \leq i \leq m, x_{i}$ and $y_{i}$ are the elements whose ladders, $R_{i}$ and $S_{i}$, fit into layer $i$. The gap-height constraint implies that the bounding boxes for $R_{i}$ and $S_{i}$ are neither above or below each other. Their widths are thus bounded by the same horizontal level of rectangles in $\mathcal{A}_{i}$. Therefore, we can assume without loss of generality that $\mathcal{A}_{i}$ is layed out as a dissection and thus by Lemma 6.1 in width $\Delta_{i}$. Because $R_{i}$ and $S_{i}$ fit into the layer- $i$ gaps, it follows that $x_{i}+y_{i}+4 \leq w(i)=2 B^{*}+4-\Delta_{i}$ and hence $x_{i}+y_{i} \leq B_{i}$. As this is true for all layers, Equation (1) implies that $x_{i}+y_{i}=B_{i}$ for all $i$, which gives a solution to $\mathcal{I}$.

Theorem 6.3 Given a graph $G$ and values $W, H, A \in \mathbb{Z}^{+}$, determining if $G$ has a (strong or weak) rectangular layout of (1) width no more than $W$ and height no more than $H$ or (2) area no more than $A$ is 


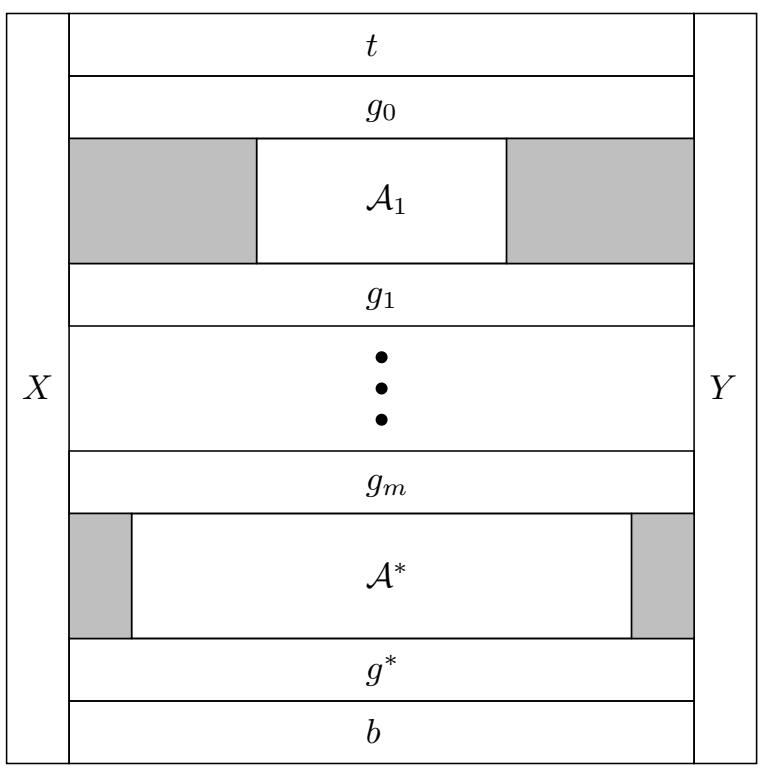

(a)

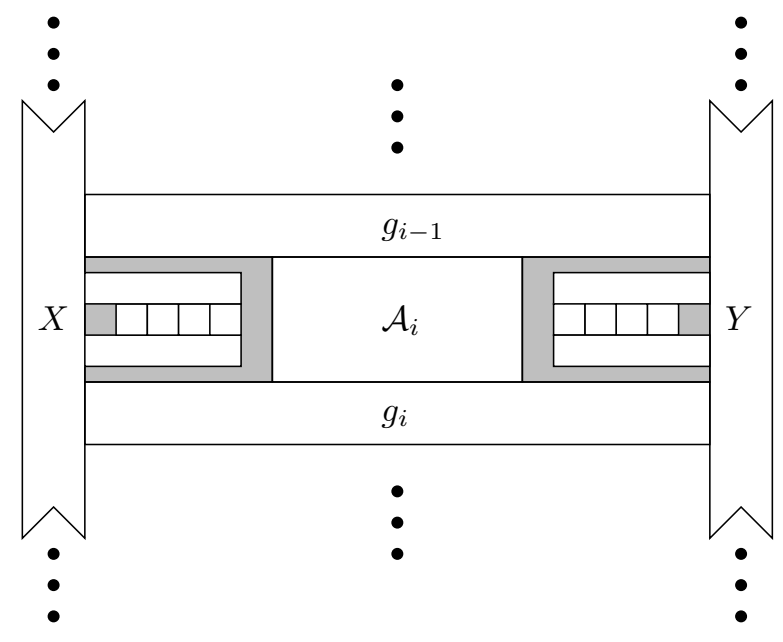

(b)

Figure 11: (a) An incompressible sub-layout for $G(\mathcal{I})$. (b) Filling the gaps in layer $i$ by some $R_{i}$ and $S_{i}$.

NP-complete.

Proof. Theorem 4.1 implies that the problem is in NP. Lemma 6.2 provides a P-time reduction showing NP-completeness, because the number of vertices and edges in $G(\mathcal{I})$ is poly $(m)$ and numerical matching with target sums is strongly NP-complete: for problem (1), set $W=2 B^{*}+6$ and $H=6 m+9$, and for problem (2), set $A=(6 m+9)\left(2 B^{*}+6\right)$. A similar reduction using height-3 accordions can be used for weak layouts.

Corollary 6.4 Given a graph $G$ and value $L \in \mathbb{Z}^{+}$, it is NP-hard to determine the minimum width (rsp., height) layout of $G$ such that the height (rsp., width) does not exceed $L$.

\subsection{Area Monotonicity}

We now explore differences between rectangular layouts and duals. First we demonstrate that weak layouts, strong layouts, and duals all have distinct area monotonicity properties. Then we show that for graphs admitting both layouts and duals, the different representations might require significantly different areas.

For any graph $G=(V, E), V^{\prime} \subset V$, and $E^{\prime} \subset E$, define $G_{V^{\prime}}$ to be the induced subgraph on $V \backslash V^{\prime}$ and $G_{E^{\prime}}$ that on $E \backslash E^{\prime}$ (removing isolated vertices). By Corollary 3.5, we know that both $G_{V^{\prime}}$ and $G_{E^{\prime}}$ have layouts if $G$ has a layout. The same does not necessarily hold for rectangular duals, however. In general, given a rendering strategy - in this case rectangular layouts or rectangular duals-we say that a vertex or edge subset is rendering preserving if the corresponding subgraph defined above admits such a rendering.

Consider monotonicity of areas under augmentation. For a given rendering strategy such that $A^{*}(G)$ is the area of an optimal rendering of $G$ (assuming $G$ admits a rendering), we say the rendering is vertex (rsp., edge) monotone if for any graph $G$ and any rendering-preserving vertex subset $V^{\prime}$ (rsp., edge subset $E^{\prime}$ ) it is true that $A^{*}\left(G_{V^{\prime}}\right) \leq A^{*}(G)\left(\right.$ rsp., $A^{*}\left(G_{E^{\prime}}\right) \leq A^{*}(G)$ ). 


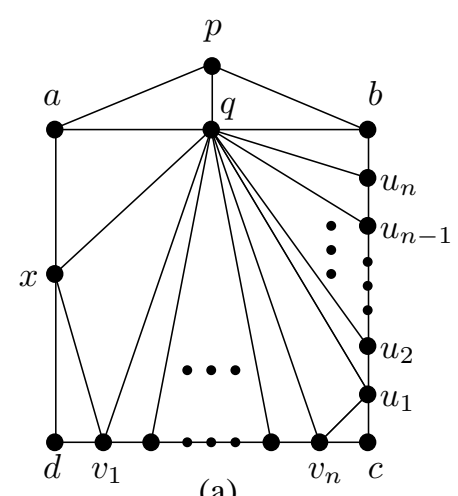

(a)

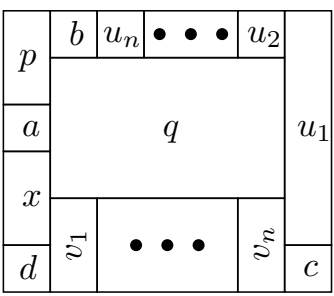

(b)

Figure 12: (a) A graph on $2 n+7$ vertices. (b) A rectangular dual of area $6(n+2)$.

\section{Theorem 6.5}

\section{Weak layouts are vertex and edge monotone.}

2. Strong layouts are vertex monotone but not edge monotone.

3. Rectangular duals are neither vertex nor edge monotone.

Proof. (1) A weak layout for $G$ is also a weak layout for any subgraph of $G$.

(2) Given a strong layout $L$ of $G$, removing the rectangle corresponding to $v$ yields a strong layout of $G_{\{v\}}$ of no greater area; hence, strong layouts are vertex monotone. Figure 1 disproves edge monotonicity, however: by inspection, any strong layout for the graph in Figure 1(a) must have area at least 9, whereas the edge-augmented graph in Figure 1 b) has an area-6 strong layout.

(3) For any $n \in \mathbb{Z}^{+}$, Figure 12 shows a graph $G$ on $2 n+7$ vertices and a rectangular dual of area $6(n+2)$. Suppose, however, we delete vertex $p$. In any rectangular dual of $G_{\{p\}}$ (as depicted in Figure 13 a)), rectangles $a, b, c$, and $d$ must be the corners of the dissection, for each has degree only 2 . The height and width must therefore each be $\Omega(n)$, as exemplified in Figure 13 b). The area is thus $\Omega\left(n^{2}\right)$, which disproves vertex monotonicity and also edge monotonicity, because the latter implies the former.

\subsection{Gaps between Layouts and Duals}

Consider the potential gap between minimum-area layouts and rectangular duals of the same graph. Define $A_{s}^{*}(G)\left(\right.$ rsp., $\left.A_{D}^{*}(G)\right)$ to be the area of an optimal strong rectangular layout (rsp., rectangular dual) of $G$.

Theorem 6.6 There exists an infinite family $\mathcal{G}$ of graphs such that for any $G \in \mathcal{G}, A_{s}^{*}(G)=O(n)$ but $A_{D}^{*}(G)=\Omega\left(n^{2}\right)$.

Proof. Define $\mathcal{G}$ to contain the graph shown in Figure 13 a) for each $n \in \mathbb{Z}^{+}$. Consider some $n \in \mathbb{Z}^{+}$and the corresponding $G \in \mathcal{G}$. As argued in the proof of Theorem 6.5, any rectangular dual of $G$ has area $\Omega\left(n^{2}\right)$, while Figure 13(c) depicts a strong rectangular layout of height 4 and width $n+2$. 


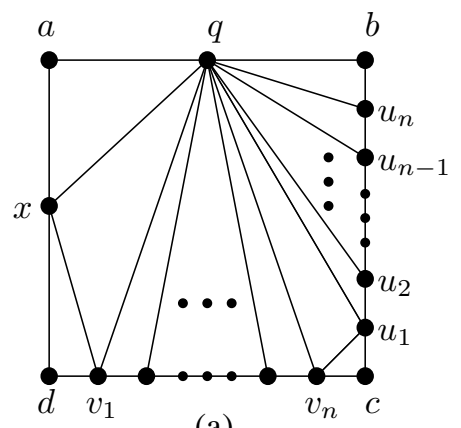

(a)

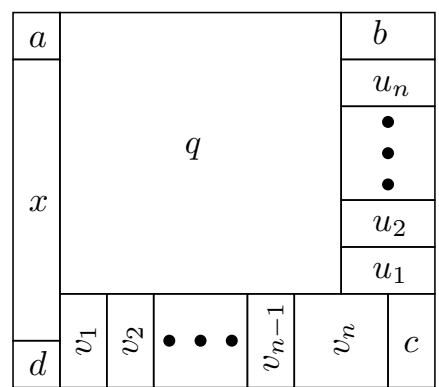

(b)

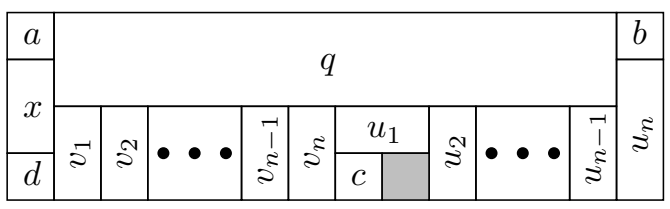

(c)

Figure 13: (a) The graph on $2 n+6$ vertices derived by deleting vertex $p$ from Figure 12(a). (b) A rectangular dual of area $(n+3)(n+2)$. (c) A strong rectangular layout of area $4(n+2)$.

\section{Conclusion}

We have presented a new characterization of contact graphs of isothetic rectangles in terms of those planar graphs that can be embedded with no filled three-cycles. We have shown the general area and constrainedwidth (and -height) optimization problems for rectangular layouts to be NP-hard and provided $O(n)$-time algorithms to construct $O\left(n^{2}\right)$-area rectangular layouts for graphs and $O(n \log n)$-area rectangular layouts for trees.

Many open problems remain. What is the hardness of approximating the minimum-area rectangular layout? Are there better approximation algorithms than the ones we presented here $(O(n)$-approximation for graphs; $O(\log n)$ for trees)? Is approximating the minimum dimension (width or height) easier than approximating the area? This problem is motivated by applications on fixed-width, scrollable displays. Also, does the NP-hardness result extend to rectangular duals? Since graphs that admit such duals are internally triangulated (triangulated except for the outer face), the freedom to place components to satisfy partition-type reductions does not seem to exist.

Can our techniques be applied to study contact graphs on other closed shapes: for example, squares, arbitrary regular polygons, arbitrary convex polygons, and higher-dimensional shapes? Also, allowing corner touching to imply adjacency changes the class of graphs described by layouts. For example, $K_{4}$ can be expressed by four rectangles meeting at a corner, and $K_{6}$, which is not planar, can be expressed by triangles. For $k \geq 4$, however, no layout on $k$-gons can express a non-planar graph. Allowing corner touching also opens the question of allowing non-isothetic rectangles, which can represent embeddings with filled triangles. Finally, is there a class of polygonal shapes other than disks whose contact graphs are the planar graphs?

\section{Acknowledgements}

We thank Therese Biedl and Chandra Chekuri for fruitful discussions and Anne Rogers for her tolerance. We thank the anonymous referees for many constructive comments. 


\section{References}

[1] A. Accornero, M. Ancona, and S. Varini. All separating triangles in a plane graph can be optimally "broken" in polynomial time. International Journal of Foundations of Computer Science, 11(3):40521, 2000.

[2] A. V. Aho, J. E. Hopcroft, and J. D. Ullman. The Design and Analysis of Computer Algorithms. Addison-Wesley, Reading, MA, 1974.

[3] G. Di Battista, P. Eades, R. Tamassia, and I. Tollis. Graph Drawing: Algorithms for the Visualization of Graphs. Prentice-Hall, Upper Saddle River, NJ, 1999.

[4] G. Di Battista, W. Lenhart, and G. Liotta. Proximity drawability: A survey. In Proc. Graph Drawing, DIMACS Int'l. Wks. (GD'94), volume 894 of Lecture Notes in Computer Science, pages 328-39. Springer-Verlag, 1994.

[5] J. Bhasker and S. Sahni. A linear algorithm to find a rectangular dual of a planar triangulated graph. Algorithmica, 3:247-78, 1988.

[6] T. Biedl, A. Bretscher, and H. Meijer. Rectangle of influence drawings of graphs without filled 3cycles. In Proc. 7th Int'l. Symp. on Graph Drawing '99, volume 1731 of Lecture Notes in Computer Science, pages 359-68. Springer-Verlag, 1999.

[7] T. Biedl, G. Kant, and M. Kaufmann. On triangulating planar graphs under the four-connectivity constraint. Algorithmica, 19:427-46, 1997.

[8] A. Brandstädt, V. B. Le, and J. P. Spinrad. Graph Classes: A Survey. SIAM Monographs on Discrete Mathematics and Applications. SIAM, Philadelphia, PA, 1999.

[9] M. de Berg, S. Carlsson, and M. H. Overmars. A general approach to dominance in the plane. Journal of Algorithms, 13(2):274-96, 1992.

[10] H. de Fraysseix, P. Ossona de Mendez, and P. Rosenstiehl. On triangle contact graphs. Combinatorics, Probability and Computing, 3:233-246, 1994.

[11] H. N. Gabow. Data structures for weighted matching and nearest common ancestors with linking. In Proc. 1st ACM-SIAM Symp. on Discrete Algorithms, pages 434-43, 1990.

[12] K. R. Gabriel and R. R. Sokal. A new statistical approach to geographical analysis. Systematic Zoology, 18:54-64, 1969.

[13] M. R. Garey and D. S. Johnson. Computers and Intractability: A Guide to the Theory of NPCompleteness. W.H. Freeman and Company, New York, 1979.

[14] D. Harel and R. E. Tarjan. Fast algorithms for finding nearest common ancestors. SIAM Journal on Computing, 13(2):338-55, 1984.

[15] X. He. On finding the rectangular duals of planar triangular graphs. SIAM Journal on Computing, 22(6):1218-26, 1993. 
[16] X. He. A simple linear time algorithm for proper box rectangular drawings of plane graphs. Journal of Algorithms, 40(1):82-101, 2001.

[17] P. Hliněný. Classes and recognition of curve contact graphs. Journal of Combinatorial Theory (B), 74(1):87-103, 1998.

[18] P. Hliněný and J. Kratochvíl. Representing graphs by disks and balls (a survey of recognitioncomplexity results). Discrete Mathematics, 229(1-3):101-24, 2001.

[19] J. W. Jaromczyk and G. T. Toussaint. Relative neighborhood graphs and their relatives. Proceedings of the IEEE, 80:1502-17, 1992.

[20] G. Kant and X. He. Regular edge labeling of 4-connected plane graphs and its applications in graph drawing problems. Theoretical Computer Science, 172:175-93, 1997.

[21] P. Koebe. Kontaktprobleme der konformen Abbildung. Berichte über die Verhand-lungen der Sächsischen, Akad. Wiss., Math.-Phys. Klass, 88:141-64, 1936.

[22] K. Koźmiński and W. Kinnen. Rectangular duals of planar graphs. Networks, 15:145-57, 1985.

[23] K. Koźmiński and W. Kinnen. Rectangular dualization and rectangular dissections. IEEE Transactions on Circuits and Systems, 35(11):1401-16, 1988.

[24] Y.-T. Lai and S. M. Leinwand. Algorithms for floorplan design via rectangular dualization. IEEE Transactions on Computer-Aided Design, 7:1278-89, 1988.

[25] Y.-T. Lai and S. M. Leinwand. A theory of rectangular dual graphs. Algorithmica, 5:467-83, 1990.

[26] A. S. LaPaugh. Algorithms for Integrated Circuit Layout: An Analytic Approach. PhD thesis, M.I.T., 1980.

[27] G. Liotta, A. Lubiw, H. Meijer, and S. H. Whitesides. The rectangle of influence drawability problem. Computational Geometry: Theory and Applications, 10:1-22, 1998.

[28] T. A. McKee and F. R. McMorris. Topics in Intersection Graph Theory. SIAM Monographs on Discrete Mathematics and Applications. SIAM, Philadelphia, PA, 1999.

[29] D. E. Muller and F. P. Preparata. Finding the intersection of two convex polyhedra. Theoretical Computer Science, 7(2):217-36, 1978.

[30] J. R. Munkres. Topology: A First Course. Prentice-Hall of India, New Delhi, 1999.

[31] M. H. Overmars and D. Wood. On rectangular visibility. Journal of Algorithms, 9(3):372-90, 1988.

[32] P. Pan, W. Shi, and C. L. Liu. Area minimization for hierarchical floorplans. Algorithmica, 15:550-71, 1996.

[33] M. Saider Rahman, S.-I. Nakano, and T. Nishizeki. Box-rectangular drawings of plane graphs. Journal of Algorithms, 37(2):363-98, 2000.

[34] M. Saidur Rahman, S.-I. Nakano, and T. Nishizeki. Rectangular grid drawings of plane graphs. Computational Geometry: Theory and Applications, 10(3):203-20, 1998. 
[35] M. Saidur Rahman, T. Nishizeki, and S. Ghosh. Rectangular drawings of planar graphs. Journal of Algorithms, 50(1):62-78, 2004.

[36] R. Ramakrishnan and J. Gehrke. Database Management Systems. McGraw-Hill, Boston, MA, 2000.

[37] R. C. Read. A new method for drawing a graph given the cyclic order of the edges at each vertex. Congressus Numerantium, 56:31-44, 1987.

[38] P. Rosenstiehl and R. E. Tarjan. Rectilinear planar layouts and bipolar orientations of planar graphs. Discrete Computational Geometry, 1:343-53, 1986.

[39] H. Sachs. Coin graphs, polyhedra, and conformal mapping. Discrete Mathematics, 134:133-8, 1994.

[40] B. Schieber and U. Vishkin. On finding lowest common ancestors: Simplification and parallelization. SIAM Journal on Computing, 17(6):1253-62, 1988.

[41] W. Shi. A fast algorithm for area minimization of slicing floorplans. IEEE Transactions on ComputerAided Design of Integrated Circuits and Systems, 15(12):1525-32, 1996.

[42] P. Steadman. Graph-theoretic representation of architectural arrangement. In L. March, editor, The Architecture of Form, pages 94-115. Cambridge University Press, London, New York, Melbourne, 1976.

[43] L. Stockmeyer. Optimal orientations of cells in slicing floorplan designs. Information and Control, 57:91-101, 1983.

[44] S. Sur-Kolay and B. B. Bhattacharya. Inherent nonslicability of rectangular duals in VLSI floorplanning. In Proc. 8th Conf. on Foundations of Software Technology and Theoretical Computer Science, volume 338 of Lecture Notes in Computer Science, pages 88-107. Springer-Verlag, 1988.

[45] S. Sur-Kolay and B. B. Bhattacharya. On the family of inherently nonslicible floorplans in VLSI layout design. In Proc. IEEE Int'l. Conf. on Circuits and Systems, volume 5, pages 2850-3, 1991.

[46] K. Tani, S. Tsukiyama, S. Shinoda, and I. Shirakawa. On area-efficient drawings of rectangular duals for VLSI floor-plan. Mathematical Programming, 52:29-43, 1991.

[47] R. E. Tarjan. Applications of path compression on balanced trees. Journal of the ACM, 26(4):690-715, 1979.

[48] C. Thomassen. Plane representations of graphs. In J. A. Bondy and U. S. R. Murty, editors, Progress in Graph Theory, pages 43-69. Academic Press, Canada, 1984.

[49] C. Thomassen. Interval representations of planar graphs. Journal of Combinatorial Theory (B), 40:920, 1988.

[50] P. Ungar. On diagrams representing maps. Journal of the London Mathematical Society, 28:336-42, 1953.

[51] D. B. West. Introduction to Graph Theory. Prentice-Hall, Upper Saddle River, NJ, 1996.

[52] H. Whitney. 2-isomorphic graphs. American Journal of Mathematics, 55:245-54, 1933. 
[53] S. Wimer, I. Koren, and I. Cederbaum. Floorplans, planar graphs, and layouts. IEEE Transactions on Circuits and Systems, 35(3):267-78, 1988.

[54] G. K. Yeap and M. Sarrafzadeh. A unified approach to floorplan sizing and enumeration. IEEE Transactions on Computer-Aided Design of Integrated Circuits and Systems, 12(12):1858-67, 1993.

[55] G. K. Yeap and M. Sarrafzadeh. Sliceable floorplanning by graph dualization. SIAM Journal on Discrete Mathematics, 8(2):258-80, 1995. 


\section{A Proofs of Lemmas}

Lemma 2.2 A graph $G$ is a 4-connected planar triangulation if and only if $G^{*}$ is planar, cubic, and cyclically 4-edge connected.

Proof. $G$ is a planar triangulation if and only if $G^{*}$ is planar and cubic. It therefore suffices to prove that (1) if $G$ is a planar triangulation that is not 4-connected, then $G^{*}$ is not cyclically 4-edge connected, and (2) if $G^{*}$ is cubic and planar but not cyclically 4-edge connected, then $G$ is not 4-connected.

Assume that $G$ is a planar triangulation that is not 4-connected. By Lemma $2.1 G$ has some separating triangle $(a, b, c)$. In $G^{*}$, therefore, there are edges $\overline{\{a, b\}}, \overline{\{b, c\}}$, and $\overline{\{c, a\}}$, each connecting the two faces in $G$ incident upon edges $\{a, b\},\{b, c\}$, and $\{c, a\}$, resp. These three edges separate $G^{*}$ into two components, $G_{1}^{*}$ and $G_{2}^{*}$. That $(a, b, c)$ is a separating triangle and $G$ is triangulated implies that $G_{1}^{*}$ and $G_{2}^{*}$ contain cycles. Hence $G^{*}$ is not cyclically 4-edge connected.

Assume that $G^{*}$ is cubic and planar but not cyclically 4-edge connected. Then there exist three edges $e_{1}, e_{2}$, and $e_{3}$ that separate $G^{*}$ into two or three components, each of which contains a cycle. Assume for now a separation into two components, $G_{1}^{*}$ and $G_{2}^{*}$. Without loss of generality, each of $e_{1}, e_{2}$, and $e_{3}$ has an endpoint in each component. Therefore there are vertices $v_{1}, v_{2}$, and $v_{3}$ in $G$ that correspond to the three faces induced in $G^{*}$ between pairs of $e_{1}, e_{2}$, and $e_{3}$. Furthermore, $\left(v_{1}, v_{2}, v_{3}\right)$ forms a separating triangle, because the cyclic nature of $G_{1}^{*}$ and $G_{2}^{*}$ implies that the corresponding components $G_{1}$ and $G_{2}$ in $G$ are non-empty. By Lemma 2.1, therefore, $G$, which is a planar triangulation, is not 4-connected. A similar argument holds when $G^{*}$ is separated into three components, in which case some pair among $e_{1}, e_{2}$, and $e_{3}$ separates $G^{*}$ into two components.

Lemma 3.1 A graph $G$ is planar, cubic, and cyclically 4-edge connected if and only if $G$ is a saturated plane map with no 3-ring.

Proof. Assume $G$ is a saturated plane map with no 3-ring. By definition, $G$ is cubic and planar, and so $G^{*}$ is a planar triangulation. Furthermore, because a 3-ring in $G$ induces a separating triangle in $G^{*}$ and vice-versa, it follows that $G^{*}$ has no separating triangle. By Lemma 2.1, therefore, $G^{*}$ is 4-connected, and by Lemma 2.2, $G$ is cubic, cyclically 4-edge connected.

Assume $G$ is planar, cubic, and cyclically 4-edge connected. Then by Lemma $2.2 G^{*}$ is a 4-connected planar triangulation. By Lemma 2.1. $G^{*}$ has no separating triangle, and so $G$ has no 3-ring. That $G$ is a saturated plane map follows the assumption by definition. 